\title{
Enhanced stability of flows through contraction channels: combining shape optimisation and linear stability analysis
}

\author{
Y. Wang, ${ }^{1, \text { a) }}$ E. Ferrer, ${ }^{2, \text { b) }}$ A. Martínez-Cava, ${ }^{2}$ Y. Zheng, ${ }^{3}$ and E. Valero ${ }^{2, b)}$ \\ 1) ETSIAE-UPM - Universidad Politécnica de Madrid - Plaza de Cardenal Cisneros 3, 28040 Madrid, \\ Spain. \\ ${ }^{2)}$ ETSIAE-UPM - Universidad Politécnica de Madrid - Plaza de Cardenal Cisneros 3, 28040 Madrid, \\ Spain \\ ${ }^{3)}$ CESC-ZJU - Centre for Engineering and Scientific Computation - Zhejiang University, Hangzhou, Zhejiang 310027 , \\ P.R. China
}

(Dated: September 27, 2019)

The first flow bifurcation, in channels with a sudden geometry contraction, is controlled through shape optimisation to delay the onset of asymmetry. First, we confirm the existence of a pitchfork type bifurcation instability, already reported in similar geometries. The global mode responsible for this bifurcation leads to asymmetric flow for Reynolds numbers beyond a critical value. Second, we propose a global shape optimisation methodology to introduce small modifications in the channel geometry that lead to flows with enhanced stability. Our results include three contraction ratios $C=2,4$ and 8 , where $C$ is the ratio of upstream to downstream channel widths.

The shape optimisation aims at minimising the growth rate of the unstable mode responsible for asymmetry. Sensitivity analysis is used to find an appropriate geometry parametrisation, which is defined through super-elliptic curves, and limited to small deformations. Additionally, a dynamically updated surrogate model (based on Radial Basis Functions) is developed to drive the optimisation. This substitutes expensive function evaluations, each requiring the solution of a steady Navier-Stokes base flow computation and a solution of an eigenvalue problem (linear stability analysis). Finally, a mode tracking algorithm identifies the eigenmode responsible for the onset of asymmetry during the optimisation.

The optimised geometries show rounded corners and are stable for Reynolds numbers well beyond the original values. For all contraction ratios, the critical Reynolds number increases by at least 7.9 times with respect to the original values. three-dimensional simulations confirm that the optimised geometry is more stable than the original when periodic boundaries are used on the side walls. When comparing the drag of the optimised geometry to the original, we obtain a reduction of at least $64 \%$.

\section{INTRODUCTION}

There have been several investigations aiming at understanding the flow behaviour in sudden contraction channels both numerically ${ }^{1-3}$ and experimentally ${ }^{4}$. In these geometries, two recirculation bubbles of different sizes are observed on each side downstream the contraction stage, once the critical Reynolds number is surpassed. Below the critical value, the flow remains symmetric. In the contraction channel, the Reynolds number $R e=D U / v$ is typically defined through the upstream channel height $D$, the mean inlet velocity $U$ and the kinematic viscosity $v$.

The experimental work of Cherdron, Durst, and Whitelaw ${ }^{5}$ and Sobey and Drazin ${ }^{6}$, reported larger recirculation regions on one side of the channel. Sobey and Drazin proposed that the asymmetry could be associated to a shear-layer instability, which would be triggered for Reynolds numbers above the the critical value. These observations were numerically replicated by Chiang and Sheu ${ }^{7}$, who showed that a supercritical pitchfork bifurcation (i.e. bifurcating from one fixed point into two fixed points ${ }^{8}$ ) is responsible for the loss of symmetry after the contraction. Once the symmetry is lost, a pressure gradient

\footnotetext{
a) Also at CESC-ZJU - Centre for Engineering and Scientific Computation Zhejiang University, Hangzhou, Zhejiang 310027, P.R. China

b) Also at CCS-UPM - Centre for Computational Simulation - Universidad Politécnica de Madrid, Boadilla del Monte, 28660 Madrid, Spain.
}

forms across the channel and leads to the well known Coanda effect $^{9}$.

Flow stability analysis ${ }^{10}$ predicts how small flow perturbations grow or decay with respect to an equilibrium flow solution (the base flow), providing information about the onset of asymmetries or unsteadiness, the physical mechanism associated to the perturbation responsible for the bifurcation, and can help to determine means by which to control it. This technique has been used to analyse similar geometries: sudden expansions ${ }^{11-14}$, sudden contractions ${ }^{7,15}$, geometries with both an expansion and a contraction ${ }^{16}, \mathrm{X}$-junctions ${ }^{17}$, or more recently T-junctions ${ }^{18-20}$. The physical mechanism behind the onset of asymmetry, in these cases, is a stationary unstable global mode. Similar global instabilities have also been reported for flows behind bumps ${ }^{21,22}$, behind backward facing steps $^{23-25}$, in boundary layer recirculation bubbles ${ }^{26}$ and in lid-driven cavities ${ }^{27,28}$.

The main objective of this work is to delay the onset of asymmetry (or increase the critical Reynolds number) in contraction channels. This task may be viewed as a passive flow control strategy, where asymmetry needs to be avoided for large values of the Reynolds number.

The linear stability community has developed tools to control the onset of bifurcations, which have been popularised as adjoint-based sensitivity analysis. Sensitivity analysis identifies the regions of the flow that, if modified, lead to the greatest damping (or amplification) of the feature responsible for the instability. The numerical study of flow sensitivity relies 
on the use of adjoint solutions ${ }^{29}$. The importance of adjoint and sensitivity maps, together with the mathematical tools required for the study of the sensitive flow regions, to different parameters, were first introduced by $\mathrm{Hill}^{30}$, and have been used extensively to examine the receptivity to internal and external modifications ${ }^{24,29,31}$, and also by the authors Ferrer, de Vicente, and Valero ${ }^{32}$, Browne et al. ${ }^{33}$, Iorio, González, and Ferrer ${ }^{34}$, Gonzalez, Ferrer, and Diaz-Ojeda ${ }^{35}$, Ferrer, Browne, and Valero ${ }^{36}$. Having determined the most sensitive flow regions, it becomes relatively simple to apply a flow control technique. Passive control introduces inactive objects (e.g. a small cylinder), which modifies the flow to stabilise or modify the frequency of particular flow features ${ }^{24}$. Useful active flow control may also be derived from sensitivity maps to control undesirable instabilities ${ }^{37}$.

Sensitivity analyses provide invaluable information of the mechanisms that control the onset of flow bifurcations. An example can be found in the recent work of Lashgari et al. ${ }^{17}$, where the sensitivity to blowing/suction was used to stabilise the onset of asymmetry in an X-junction. Applying steady blowing, they could delay the first bifurcation from $R e=82.5$ to 150 (i.e. $82 \%$ higher).

Sensitivity analysis relies on linear modal flow structures and hence are strictly valid under linear assumptions (e.g. small variations from equilibrium). For example, if one applies sensitivity to geometrical modifications, it is necessary to assume that these modification do not alter significantly the base flow, used to obtain the modal structures. For large modifications, the base flow changes and the sensitivities may not effectively guide the flow control design. An alternative to sensitivity analyses is to use a full optimisation cycle, where the modal eigenvalues constitute the objective function, and the associated base flow is recomputed at each function evaluation. In what follows, we consider the growth rates of the modes of interest as the target function and attempt to damp these through geometric variations (i.e. shape optimisation). The results are analysed and assessed using linear stability analysis and sensitivity analysis.

In recent years, aerodynamic shape optimisation has become a valuable tool for the design of efficient lifting surfaces and even entire aircraft ${ }^{38}$. The advances in computing power and development of more accurate computational fluid dynamics solvers have promoted optimal shape design ${ }^{39}$. In most recent research, Pralits and Hanifi ${ }^{40}$, Flinois and Colonius $^{41}$ and Xiao and Papadakis ${ }^{42}$ have successfully applied aerodynamic optimisation to optimal flow control stragies. However, the use of shape optimisation to delay the onset of unsteadiness has remained elusive, even for simple flows. One of the reasons is that every function evaluation requires two steps. First, a steady base flow (steady Navier-Stokes base flow simulation) needs to be obtained and, second, an eigenvalue system needs to be solved to find the stability of perturbations. The combination of these two steps leads to function evaluations that can become prohibitive. In this work, we adopt a surrogate model based on Radial Basis Functions $(\mathrm{RBF})^{43}$ to reduce the computational cost. The resulting optimisation methodology is able to find stable flows by finding the optimal shape. To the authors' knowledge, this methodol-

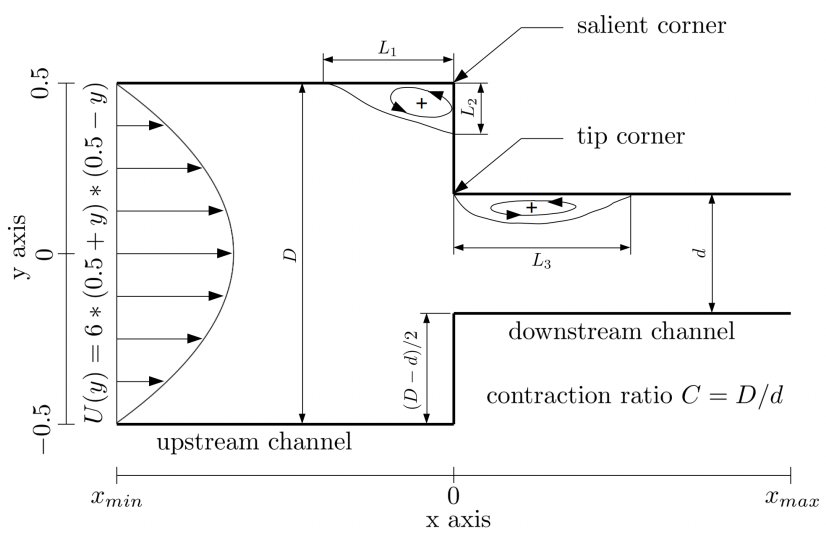

Figure 1: Geometry and the characteristic lengths.

ogy has not been presented before to enhanced flow stability.

In summary, we introduce a shape optimisation methodology that tracks the asymmetric mode and modifies the shape of the contraction channel to enhance stability. The resulting geometries are stable at large Reynolds numbers. Three main ingredients constitute our optimisation methodology. First, a geometry parametrisation is defined through super-elliptic curves. Second, a dynamically updated surrogate model (based on Radial Basis Functions) is developed to drive the optimisation investigation and substitutes expensive function evaluations. Third, a mode tracking scheme based on feature identification is developed to track the eigenmode responsible for the onset of asymmetry.

We will demonstrate that the optimised geometries shows rounded corners and are stable for critical Reynolds numbers 7.9 and 20.9 times larger than originally, for contraction ratios $C=2$ and 4, where $C$ is the ratio of upstream to downstream channel widths. The tip corner bubbles are entirely eliminated for $C=8$ for Reynolds numbers as high as $4 \times 10^{6}$. Throughout the paper, we use linear stability and flow sensitivity to analyse and discuss the results. Additionally, we validate the optmised shapes obtained in two-dimensional optimisation in three dimensions to show the effect of stabilisation by the modification of the shape in three-dimensional flow.

The remaining sections of this paper are organised as follows. Section II describes the problem and the geometry of the contraction channel. Section III includes stability and sensitivity analyses, in which, the methodology is first introduced, and then a bifurcation study is provided with various contraction ratios and Reynolds numbers. Following, section IV details the optimisation procedure and discusses the optimised shapes. Concluding remarks can be found in section V.

\section{PROBLEM DESCRIPTION}

The geometry of contraction channel is depicted in Fig. 1. A parabolic horizontal velocity profile is imposed in the left inlet, with the form: $U(y)=6 \times(0.5+y) \times(0.5-y)$. As shown in the figure, $D$ and $d$ are the widths of the channels upstream and downstream the contraction, respectively. 
Table I: Geometry parameters and separation/reattachment lengths.

\begin{tabular}{llc}
\hline \hline Name & Representation & Value \\
\hline$D$ & height of upstream channel & $1.00 \mathrm{~m}$ \\
$C$ & contraction ratio & $2,4,8$ \\
$d$ & height of downstream channel & $D / C$ \\
$x_{\max }-0$ & length of downstream channel & $5.00 \mathrm{~m}$ \\
$0-x_{\min }$ & length of upstream channel & $2.50 \mathrm{~m}$ \\
$L_{1}$ & separation length of salient corner bubble & \\
$L_{2}$ & reattachment length of salient corner bubble & \\
$L_{3}$ & reattachment length of tip corner bubble & \\
\hline \hline
\end{tabular}

Three contraction ratios will be subsequently considered: $C=$ $D / d=2,4$ and 8 . Additionally, $\left(0-x_{\min }\right)$ and $\left(x_{\max }-0\right)$ are the lengths of upstream and downstream channels. The flow in this relatively simple geometry may be characterised by two separation regions ${ }^{5-7}$. The first at the upstream salient corner (before the contraction) and the second after the contraction and close to the tip corner. The second recirculation is sometimes referred to as separation bubble. Three geometric lengths define these two recirculation regions: $L_{1}$ is the separation length of the salient corner bubble, $L_{2}$ and $L_{3}$ are the reattachment lengths of the salient corner bubble and the tip corner bubble respectively, which are defined as the distance between the separation point and the reattachment points on the walls.

A summary of the parameters describing the geometry and the flow in the contraction channel are shown in Table I. Finally, let us recall that the Reynolds number $R e=D U / v$ is typically defined, e.g. Ref. 7, through the upstream channel height $D$, the mean inlet velocity $U$ and the kinematic viscosity $v$.

\section{STABILITY AND SENSITIVITY ANALYSIS}

In this section, we consider the stability and sensitivity of the three contraction channels (with contraction ratios $C=2,4$ and 8 ) and a variety of Reynolds numbers. We first introduce the methodology for stability and sensitivity analyses to provide bifurcation diagrams, stability results and sensitivities that will subsequently be used to define the optimisation problem (in section IV).

\section{A. Methodology}

\section{Linear Stability Analysis}

We consider a discrete approach to derive sensitivity fields ${ }^{33,44}$. The discrete method starts by discretising the governing equations to subsequently derive the linearised system; as opposed to first linearising to then discretise (i.e. continuous approach). An advantage of the discrete version is that, being purely algebraic, it does not require the derivation of adjoint equations or adjoint boundary conditions since these are automatically included in the matrix system. However, we note that both continuous and discrete formulations should provide analogous results. Comparisons between discrete and continuous approaches may be found elsewhere ${ }^{29,45}$. In addition, we note that the stability analysis presented here does not assume any parallel or weakly parallel flow assumption as it is of global type.

The methodology may be implemented in compressible or incompressible flow solvers. Here we select, without loss of generality, a compressible version of the Navier-Stokes equations, where conservative variables are utilised: $\mathbf{q}=$ $(\rho, \rho u, \rho v ; \rho w ; \rho e)^{T}$, where $\rho$ denotes the density, $u, v$ and $w$ are the velocity components and $e$ denotes energy. We start by considering a spatially discretised time varying and nondimensional system

$$
\mathscr{M} \frac{\partial \boldsymbol{q}}{\partial t}=\mathscr{F}(\boldsymbol{q})
$$

where $\mathscr{F}(\boldsymbol{q})$ is a discrete non-linear operator, $\mathscr{M}$ is the mass matrix resulting from the spatial discretisation. Specific details of the finite volume formulation retained for the solution of this system are provided in section III A 4.

To perform linear instability analysis the state variable $\boldsymbol{q}$ is decomposed into its steady state contribution, $\bar{q}$, and a small perturbation, $\boldsymbol{q}^{\prime}$ such that $\boldsymbol{q}=\overline{\boldsymbol{q}}+\boldsymbol{q}^{\prime}$. We proceed by inserting this decomposition into Eq. (1) and linearise the nonlinear discrete system using Taylor series (around $\overline{\boldsymbol{q}}$ ). Subtracting the base flow equation and neglecting second order terms $\mathscr{O}\left(\boldsymbol{q}^{\prime 2}\right)$, we obtain a linearised and time varying system for the perturbation field

$$
\mathscr{M} \frac{\partial \boldsymbol{q}^{\prime}}{\partial t}=\mathscr{J}(\overline{\boldsymbol{q}}) \boldsymbol{q}^{\prime}
$$

with $\mathscr{J}(\overline{\boldsymbol{q}})=\left.\frac{\partial \mathscr{F}(\boldsymbol{q})}{\partial \boldsymbol{q}}\right|_{\overline{\boldsymbol{q}}}$ denoting the Jacobian matrix. We include a detailed derivation in Appendix A of this text. Eq. (2) may be advanced in time to simulate the growth or decay of perturbation upon the base flow $\overline{\boldsymbol{q}}^{32,46}$. Alternatively, it is possible to solve the above perturbation equation in the frequency domain by introducing the ansatz, $\boldsymbol{q}^{\prime}=\hat{\boldsymbol{q}} e^{\sigma t}$, which leads to a generalised eigenvalue problem

$$
\mathscr{J}(\overline{\boldsymbol{q}}) \hat{\boldsymbol{q}}=\sigma \mathscr{M} \hat{\boldsymbol{q}} .
$$

The eigenvector $\hat{\boldsymbol{q}}$ is the direct mode associated to the complex eigenvalue, $\sigma=\sigma_{R}+i \sigma_{I}$, whose real and imaginary components represent the perturbation growth rate and angular frequency.

\section{Discrete Adjoints}

We define a discrete adjoint eigenvalue problem by selecting the $L_{2}$ inner product in the computational domain $\Omega$, such that $\langle\tilde{\mathbf{c}}, \tilde{\mathbf{d}}\rangle_{\Omega}=\mathbf{c}^{H} \mathscr{M} \mathbf{d}$, for $\tilde{\mathbf{c}}$ and $\tilde{\mathbf{d}}$ continuous vectors associated to their discrete versions $\mathbf{c}$ and $\mathbf{d}$, where $\mathscr{M}$ is again the mass matrix (as in Eq. (1) or (3)) and $\left(\bullet^{H}\right)$ denotes the Hermitian transpose. 
Then, the discrete adjoint Jacobian matrix $\mathscr{J}^{+}$follows $\langle\mathscr{J} \tilde{\boldsymbol{c}}, \tilde{\boldsymbol{d}}\rangle_{\Omega}=\left\langle\tilde{\boldsymbol{c}}, \mathscr{J}^{+} \tilde{\boldsymbol{d}}\right\rangle_{\Omega}$. Expanding this expression and using the definition for the discrete inner product, it is easy to see that the discrete adjoint Jacobian matrix is $\mathscr{J}^{+}=$ $\mathscr{M}^{-\mathbf{1}} \mathscr{J}^{\mathbf{H}} \mathscr{M}$. In our case, the Jacobian is real and $\mathscr{J}^{\mathbf{H}}=$ $\mathscr{J}^{\mathbf{T}}$. In summary, the adjoint modes can be obtained by solving $\mathscr{J}^{+}(\overline{\boldsymbol{q}}) \hat{\boldsymbol{q}}^{+}=\sigma^{+} \mathscr{M} \hat{\boldsymbol{q}}^{+}$, with $\mathscr{J}^{+}=\mathscr{M}^{-\mathbf{1}} \mathscr{J}^{\mathbf{T}} \mathscr{M}$.

\section{Structural Sensitivity}

The localised regions of sensitivity denote the spatial separation between direct and adjoint modes, which is related to the non-normality ${ }^{47}$ of the Navier-Stokes equations. The regions where the direct and adjoint modes overlap define the structural sensitivity to localised feedback or wavemaker regions ${ }^{31}$, and relates to the origin of absolute instabilities. These sensitivity maps provide information on the flow regions where a generic force-velocity coupling causes the largest drift in the eigenvalues and hence provides useful information on control strategies to attenuate these instabilities $^{29,31,33}$. Other flow sensitivities, e.g. to base flow modification or steady forcing $33,36,44,48,49$, have been defined in the literature but are not considered here. Generally speaking, sensitivity analyses define the "sweet spots" for the location of passive control mechanisms and the regions where small modifications lead to the largest modification of the eigenmode behaviour (e.g. enhancing its stability).

The structural sensitivity maps can be calculated using the expression $S=\left\|\hat{\mathbf{q}}^{+}\right\| \cdot\|\hat{\mathbf{q}}\|$ with $<\hat{\mathbf{q}}^{+}, \hat{\mathbf{q}}>=1$, where $\hat{\mathbf{q}}$ and $\hat{\mathbf{q}}^{+}$are the direct and adjoint modes, where $\langle\bullet, \bullet\rangle$ denotes the $L_{2}$ inner product, with its associated norm $\|\bullet\|=<$ $\bullet, \bullet>^{1 / 2}$.

\section{Summary of the Methodology and Implementation Details}

a. Summary of stability and sensitivity analysis: The methodology to perform stability and sensitivity analysis can be summarised in the following 4 steps:

1. Compute a steady base flow using a numerical solver. Here, we time march the compressible Navier-Stokes equations (Eq. (1)), until the residual falls below $10^{-6}$.

2. We construct the Jacobian matrix, Eq. (2) and solve the eigenvalue problem Eq. (3).

3. Having computed all direct eigenvalues/eigenmodes, we select the least stable and compute its adjoint (see section III A 2).

4. With the direct and adjoint modes, it is possible to compute the structural sensitivity, as detailed in section III A 3.

The resulting sensitivities guide the design of the shape optimisation, which is detailed in section IV. b. Flow simulations and spatial discretisation: In the present investigation, we use DLR-TAU code for the simulation. The DLR-TAU code is a compressible three-dimensional Navier-Stokes solver. In this work, we only consider the laminar version of the solver and unstructured meshes.

Spatial discretisation uses a finite volume approach. In this formulation the mass matrix $\mathscr{M}=\int_{\Omega} d V$ simplifies to the computational cell volumes $\Omega$.

When the compressible Navier-Stokes equations are selected, the right hand side $\mathscr{F}(\boldsymbol{q})$ in (1) denotes the divergence of the viscous and convective fluxes:

$$
\begin{aligned}
\mathscr{F}(\mathbf{q}) & =\int_{\Omega} \nabla \cdot\left(\boldsymbol{F}_{v}-\boldsymbol{F}_{e}\right) \\
& =\int_{\partial \Omega}\left(\boldsymbol{F}_{v}-\boldsymbol{F}_{e}\right) \cdot \mathbf{n},
\end{aligned}
$$

where $\boldsymbol{F}_{e}$ are the inviscid, or Euler fluxes, and we have used using Gauss' divergence theorem to replace the divergence of the fluxes by the fluxes through the element faces $\partial \Omega$. Convective fluxes are discretised using a second order Van Leer Upwind scheme.

$$
\boldsymbol{F}_{e}=\left[\begin{array}{ccc}
\rho u & \rho v & \rho w \\
\rho u^{2}+p & \rho u v & \rho u w \\
\rho u v & \rho v^{2}+p & \rho v w \\
\rho u w & \rho v w & \rho w^{2}+p \\
\rho u H & \rho v H & \rho w H
\end{array}\right]
$$

where $\rho$ and $H$ are the density and total enthalpy. Additionally, $\boldsymbol{F}_{v}$ defines the viscous fluxes:

$\overrightarrow{\boldsymbol{F}}_{v}=\left[\begin{array}{ccc}0 & 0 & 0 \\ \tau_{x x} & \tau_{x y} & \tau_{x z} \\ \tau_{y x} & \tau_{y y} & \tau_{y z} \\ \tau_{z x} & \tau_{z y} & \tau_{z z} \\ \sum_{j=1}^{3} v_{j} \tau_{1 j}+\kappa T_{x} & \sum_{j=1}^{3} v_{j} \tau_{2 j}+\kappa T_{y} & \sum_{j=1}^{3} v_{j} \tau_{3 j}+\kappa T_{z}\end{array}\right]$

where $\kappa$ is the thermal conductivity, $T_{x}, T_{y}$ and $T_{z}$ denote the gradients of temperature and the stress tensor $\tau$ is defined as $\boldsymbol{\tau}=\mu\left(\nabla \vec{v}+(\nabla \vec{v})^{T}\right)-2 / 3 \mu \boldsymbol{I} \nabla \cdot \vec{v}$, with $\mu$ the dynamic viscosity, $\vec{v}=(u, v, w)^{T}$ and $\boldsymbol{I}$ is the three-dimensional identity matrix. In all cases, the Mach number is kept low such that compressible effects are minimum.

Regarding boundary conditions, Dirichlet boundary data is imposed at inflow, whereas exit pressure flow condition is imposed after the contraction. No-slip boundary conditions are used for the upper and lower boundaries. Table II summarises these boundary conditions.

c. Flow simulations and time evolution: For both stable and unstable configurations (resp. symmetrical and asymmetric), base flow solutions are obtained marching in time towards steady state by a backward Euler implicit scheme, solved approximately by a LU-SGS (Lower-Upper Symmetric-Gauss-Seidel Method) iterations procedure. Local time stepping and multigrid algorithms are adopted to accelerate convergence. The convergence criteria is set such that the 
Table II: Contraction channel boundary conditions.

\begin{tabular}{ccc}
\hline \hline Boundary name & Boundary condition & Notes \\
Inlet & Dirichlet & $V(y)=6 *(0.5+y) *(0.5-y)$ \\
Outlet & Exit pressure flow & \\
Wall & No-slip \\
\hline \hline
\end{tabular}

global density residual is below $10^{-6}$. Steady state solutions are obtained by solving the base flow only for half domain, which is subsequently mirrored to perform the stability analysis in a full domain.

d. Stability analyses: To perform stability analyses, a steady solution, known as base flow, is required. For $R e<$ $R e_{c r i}$, we can simulate a half domain or a full domain (both providing identical results) to obtain stable symmetric base flows. However, for $R e>R e_{c r i}$ a stable asymmetric solution naturally develops in a full domain. Hence to obtain a stable base flow for stability analysis, a steady base flow with only half domain is computed (using a slip-wall boundary condition on the symmetry plane) and mirrored about the centre line, to then perform stability analysis on the full domain. Note that above the $R e_{c r i}$, the steady solution with the full domain converges to asymmetric flow patterns. The resulting technique forces a symmetric base flow, enabling the calculation of unstable asymmetric modes.

In summary, to compute symmetric unstable base flows as:

1. Converge a steady state half domain simulation.

2. Mirror the half domain to conform a full domain.

3. Perform the stability analysis using the full domain flow (i.e. the forced symmetric unstable flow), which results in unsteady eigenvalues (showing the instability of the flow created through mirroring).

The eigenvalue problem, (3), is solved by full LU factorisation performed on the Jacobian matrix using the MUMPS package $^{50}$, followed by an Arnoldi algorithm with shift-andinvert transformation to obtain the eigenvalues of a reduced Krylov subspace throughout LAPACK ${ }^{51}$ routines. The eigenvalues of the reduced system approximate some of the eigenvalues of the original eigenvalue problem, and can be controlled through the shift-and-invert transformation. This approach has already been used by the authors in Refs. 32-35.

\section{Convergence Study}

We first include a grid convergence study for the steady base flows, based on the recirculation regions $\left(L_{1}, L_{2}\right.$ and $L_{3}$ ) defined in section II. We select two Reynolds numbers $R e=1000$ and 2000 and a contraction ratio $C=2$, since these conditions enable comparisons with previously published results by Dennis and Smith ${ }^{1}$, Hunt ${ }^{2}$, Hawken, Townsend, and Webster $^{3}$, Chiang and Sheu ${ }^{7}$. Results are summarised in Table III. We consider that grid independence is achieved, when the results of two successive grids are smaller than 5 percent,
Table III: Grid convergence: Separation and reattachment lengths $L_{1}, L_{2}$ and $L_{3}$ for contraction ratio $C=2$.

\begin{tabular}{lccccccc}
\hline \hline \multicolumn{1}{c}{ Length } & \multicolumn{4}{c}{$L_{1}$} & \multicolumn{2}{c}{$L_{2}$} & \multicolumn{2}{c}{$L_{3}$} \\
\hline Reynolds number & 1000 & 2000 & 1000 & 2000 & 1000 & 200 \\
\hline Dennis and Smith $^{1}$ & 0.138 & 0.182 & 0.081 & 0.093 & & \\
Hunt $^{2}$ & 0.154 & 0.197 & 0.82 & 0.094 & 0.207 & 0.481 \\
Hauken et al. $^{3}$ & 0.143 & 0.077 & & 0.239 & \\
Chiang and Sheu $^{7}$ & 0.140 & 0.183 & 0.082 & 0.094 & 0.222 & 0.543 \\
Present: $160 \times 80, \mathrm{~h}=1 / 80$ & 0 & 0.062 & 0 & 0.006 & 0 & 0 \\
$320 \times 160, \mathrm{~h}=1 / 160$ & 0.031 & 0.031 & 0.013 & 0.018 & 0.060 & 0.241 \\
$\quad 640 \times 320, \mathrm{~h}=1 / 320$ & 0.143 & 0.185 & 0.063 & 0.068 & 0.182 & 0.453 \\
$1280 \times 640, \mathrm{~h}=1 / 640$ & 0.143 & 0.187 & 0.079 & 0.089 & 0.239 & 0.548 \\
$2560 \times 1280, \mathrm{~h}=1 / 1280$ & 0.144 & 0.193 & 0.081 & 0.092 & 0.242 & 0.550 \\
$\quad$ locally refined & 0.143 & 0.189 & 0.081 & 0.090 & 0.241 & 0.550 \\
\hline \hline
\end{tabular}

Table IV: Detailed parameters of the locally refined mesh.

\begin{tabular}{ccccc}
\hline \hline$C$ & $N_{\Delta x}$ & $N_{\Delta y}$ & $\left(\Delta x_{\min }, \Delta x_{\max }\right)$ & $\left(\Delta y_{\min }, \Delta y_{\max }\right)$ \\
\hline $2,4,8$ & 320 & 360 & $(0.001,0.012)$ & $(0.0005,0.003)$ \\
\hline \hline
\end{tabular}

for the $L_{1}, L_{2}$ and $L_{3}$ lengths. The biggest difference in lengths between the last two sets of grids is 2 percent, so the results we obtain from the grid $h=1 / 1280(2560 \times 1280$ elements $)$ can be considered independent of the mesh. This last mesh has 2 million elements, which leads to very costly computations. Instead of using homogeneous mesh refinement, it is more efficient to use a comparatively coarse mesh with local refinements, where mesh points are clustered near the channel corners. The results from the locally refined mesh are shown in comparison with uniform meshes in Table III. By using the locally refined mesh, the computation time is reduced to only 5 percent of that of the finest uniform mesh, while the accuracy is comparable. The final parameters used for the locally refined mesh are included in Table IV. Additionally, let us note that the converged results show good agreement with previous published data.

Having checked the convergence of the base flows, we now proceed to inspect the convergence of the eigenvalues. To this aim, we compare the eigenvalues for the asymmetric mode at $R e=2500$ and 4000 for $C=2$, in Table V. We select higher Reynolds ( $R e=2500$ and 4000) since these represent a symmetric (stable) and an asymmetric (unstable) state for the contraction ratio $C=2$, respectively. As for the base flow, convergence is obtained for the grid $h=1 / 1280(2560 \times 1280$ elements). The eigenvalues obtained for the locally refined mesh are identical (with the retained accuracy of $10^{-3}$ ) to the finest homogeneous mesh.

Finally, retaining the locally refined mesh, we analyse the influence of the Krylov space size in the convergence of the eigenvalues, when using the Arnoldi method. The Krylov subspace dimension controls the accuracy of the eigenvalues for the Arnoldi methods. Table VI shows that a Krylov space if dimension 160 is sufficient to obtain accurate eigenvalues for the two Reynolds numbers. Finally, note that to obtain the previous eigenvalue convergence for grid refinement, Table V, 
Table V: Grid convergence fro eigenvalues: real part $\left(\sigma_{R}\right)$ of the most unstable asymmetric mode, for contraction ratio

$$
C=2 \text {. }
$$

\begin{tabular}{lccc}
\hline \hline Reynolds number & & 2500 & 4000 \\
\hline uniform: & $40 \times 20$ & -0.193 & -0.142 \\
& $80 \times 40$ & -0.241 & -0.173 \\
& $160 \times 80$ & -0.197 & -0.094 \\
& $320 \times 160$ & -0.205 & 1.378 \\
& $640 \times 320$ & -0.253 & 1.623 \\
& $1280 \times 640$ & -0.294 & 1.763 \\
& $2560 \times 1280$ & -0.294 & 1.764 \\
locally refined & & -0.294 & 1.764 \\
\hline \hline
\end{tabular}

Table VI: Krylov subspace dimensions convergence for eigenvalues: Real part $\left(\sigma_{R}\right)$ of the most unstable asymmetric mode, for contraction ratio $C=2$.

\begin{tabular}{llcc}
\hline \hline Reynolds number & & 2500 & 4000 \\
\hline Krylov & 40 & -0.293 & 1.437 \\
dimension: & 80 & -0.294 & 1.734 \\
& 160 & -0.294 & 1.764 \\
& 320 & -0.294 & 1.764 \\
\hline \hline
\end{tabular}

we selected 320 to avoid Krylov dimensions effects.

In summary, the results included in subsequent sections use local refinement (see Table IV) and Krylov spaces of size 320.

\section{B. Base Flows and Onset of Asymmetries}

We select the recirculation length $L_{3}$ as the criterion to determine when flow symmetry is lost and the flow bifurcates following a supercritical pitchfork bifurcation, see figure 2 . We depict the $L_{3}$ bubble size against Reynolds numbers for contraction ratios $C=2,4$ and 8 , in Fig. 2. Note that due to the symmetry of the channel, the large bubble can appear near the upper or the lower corner randomly and consequently, we choose the same symbol to show the sizes of bubbles when considering the full domain simulations in Fig. 2. Additionally, when the Reynolds number is below the critical value, the two curves overlap. The critical Reynolds number where the flow bifurcates and asymmetries appear decreases for increasing contraction ratios. We obtain critical Reynolds: $R e_{c r i}=3150$ for $C=2, R e_{c r i}=1420$ for $C=4$ and $R e_{c r i}=1230$ for $C=8$. Similar bifurcation diagrams and critical Reynolds numbers were reported by Chiang and Sheu ${ }^{7}$.

Additionally, Fig. 2 shows that the reattachment lengths of the two recirculation bubbles (at both sides of the contraction) become larger as the Reynolds number increases. Lashgari et al. ${ }^{17}$ suggested that the point of the bifurcation is determined by the size of the recirculation region, in a $\mathrm{X}$-junction flow. However, in our contraction channel, the size of the recirculation region at the critical Reynolds number shows a nonlinear decrease for increasing contraction ratios. This may be seen in Fig. 2, where the approximate bubble sizes

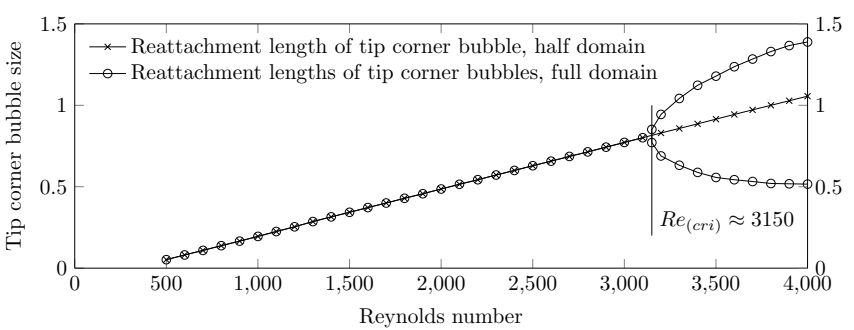

(a) $C=2$.

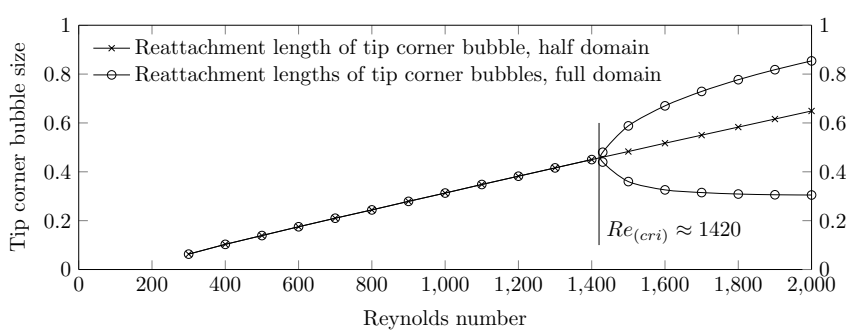

(b) $C=4$.

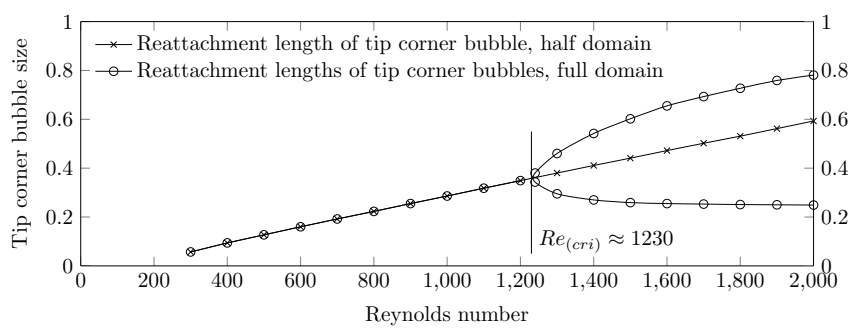

(c) $C=8$.

Figure 2: Bifurcation diagram for contraction ratios $C=2,4$ and 8, illustrated using the reattachment lengths $L_{3}$ of downstream tip corner eddy for varying Reynolds numbers.

are 0.8 for $C=2,0.5$ for $C=4$ and 0.4 for $C=8$. In both configurations nonetheless, the mechanism responsible for bifurcation is comparable. As the recirculation bubbles grow in size, the contraction becomes effectively smaller, such that the side bubbles approach, which facilitates the vertical momentum transfer. This mechanism will be further explored in following sections and controlled through the proposed shape optimisation.

We illustrate the symmetric and asymmetric flows in Fig. 3 , where we present results for Reynolds numbers 1000 and 2000 for contraction ratio $C=4$. For Reynolds number 1000 , the simulation converges to a symmetric flow, see Figs. $3 \mathrm{a}$ and $3 c$. Two recirculation bubbles of the same size form downstream of the contraction and near the tip corners. Further downstream, due to the viscous dissipation, the flow regains its Poiseuille channel flow profile. When the Reynolds number is increased to 2000, an asymmetric steady flow develops, see Figs. 3b and 3d. In the asymmetric case, two recirculation bubbles of unequal size develop and lead to a lost of symmetry and the well reported Coanda effect ${ }^{9}$. Figs. 3c and $3 \mathrm{~d}$ detail the recirculation regions. The Coanda effect is a consequence of the pressure difference established in the cross-stream direction ${ }^{6}$. Note that the new asymmetric flow 
is steady and no periodic flow develops, confirming that the bifurcation is of pitchfork type.

Hawa and Rusak ${ }^{52}$ suggested that asymmetries resulted from the interaction between the destabilising upstream convection effects by the asymmetric perturbation and the combined stabilising effects of the viscous dissipation and the downstream convection of perturbations by the base symmetric flow. However, Chiang and Sheu ${ }^{7}$ and Lashgari et al. ${ }^{17}$ have shown that an absolute instability is responsible for the pitchfork bifurcation resulting in lost of symmetry. This will be confirmed in the next section through linear stability analyses.

Finally and for completeness, we depict in Fig. 4 the recirculation regions for various Reynolds numbers. It can be seen that the bubble becomes larger when the Reynolds number increases. Additionally, we observe that for asymmetric unstable flows, unequal bubbles develop.

\section{Stability Analysis}

To better understand the nature of the presented bifurcations, we conduct linear stability analyses for Reynolds number ranges $R e=2500$ to $4000, R e=900$ to 2000 and $R e=900$ to 2000 , for contraction ratios $C=2,4$ and 8 , respectively. In Fig. 5, we present the evolution of the growth rates (or real part of the eigenvalue) of the least stable or unstable eigenvalue, against the Reynolds number. We include the critical Reynolds number at which the eigenvalue crosses the real axis, indicating the onset of instability. By comparing these results to the previous bifurcation diagrams (see Fig. 2) in section III B, it is clear that the bifurcations start at exactly the same Reynolds numbers, which indicates that the selected eigenvalues govern the onset of asymmetry.

Additionally, Figs. $6 \mathrm{a}$ and $6 \mathrm{~b}$ illustrate the eigenvalues for contraction ratio $C=4$ and Reynolds numbers $R e=1000$ and 2000, and mark the least stable asymmetric mode with a filled square. We observe that for $R e=1000$ all eigenvalues have negative real part, which indicates that the flow is stable, whilst for $R e=2000$, there is one unstable mode (positive real part), which is obtained by analysing the symmetric unstable flow obtained through mirroring half domain. Note that all modes are complex but the modes without imaginary component (e.g. asymmetric modes) represent flow structures without temporal oscillating frequencies.

The eigenmodes corresponding to the marked eigenvalues (filled squares) are depicted in Fig. 6. Particularly, we depict the component of the eigenmode corresponding to the streamwise velocity in Figs. $7 \mathrm{a}$ and $7 \mathrm{~b}$.We observe that for both Reynolds numbers, the eigenmodes are asymmetric. These modes are responsible for the first bifurcation and the loss of symmetry. The $u$ perturbation, shown in Figs. 7a and 7b, is responsible for the increased speed at the outer edge of the top bubble and the decrease at the outer edge of the bottom bubble. The vertical velocity component perturbation (v) (not shown) modifies the flow upwards at $x=0.2$ and downwards at $x=0.7$. The structure of these global modes shows that the streamwise acceleration leads to lower pressure at the up- per recirculation area, and higher pressure at the lower recirculation area, which in turn induces a cross-stream pressure gradient that helps to maintain the asymmetric flow profile.

In addition, Figs. 7c and 7d show the adjoint modes corresponding to the previous direct modes. It is noticeable that the adjoint modes are located near the salient corner and the tip corner, denoting the importance of these corners in the receptivity of the direct modes and the stability of the flow.

\section{Structural Sensitivity}

The regions with highest structural sensitivity (see section III A 3) indicate the zones where a localised modification will lead to significant changes in the mode and will need to be considered to control the flow. This sensitivity map is obtained by overlapping the direct and adjoint global modes, as shown in Fig. 8, where the recirculation line (in red) has also been included for reference.

The highest structural sensitivity appears near the edge of the recirculation bubbles and extends from the contraction corner. This shape suggests that by manipulating the geometry of the corner and the surrounding region, the instability may be controlled.

The spatial map of the production of the perturbation kinetic energy is shown in Fig. $8 b$ for the same flow, and has been normalised by the overall perturbation kinetic energy in the domain. We found that the region with largest transfer of the kinetic energy from the base state to the perturbations are located in the shear layer at the edge of the recirculation bubbles, and the core of the instability is found to be near the region with maximum positive and negative production. More specifically, we compute the contribution of the lift-up perturbation kinetic energy term: $u v d U / d y$, shown in Fig. 8c. Comparing Figs. $8 \mathrm{~b}$ and $8 \mathrm{c}$, we can see that the energy production by the term $u v d U / d y$ and the total energy production are almost identical in shape and amplitude, confirming that a lift-up mechanism is responsible for the onset of asymmetry. Similar conclusions have been reported by ${ }^{17}$ for an $\mathrm{X}$-junction geometry. The lift-up mechanism, where the disturbance extracts energy from the base flow through the term $u v d U / d y$, is common in several shear flow instabilities such as Kelvin-Helmholtz instabilities or Tollmien - Schlichting waves. Further details may be found elsewhere ${ }^{53}$.

In summary, we have reproduced published results to verify that the onset of asymmetry is governed by an absolute instability (an asymmetric mode). When computing the adjoint of this mode and associated structural sensitivity, we observe a region of high sensitivity near the contraction, starting at the corner and extending downstream. In what follows, we will parametrise the geometry to enable a shape optimisation that can modify the geometry of the sharp corners (by a small amount), to enhance the stability of the problem. 


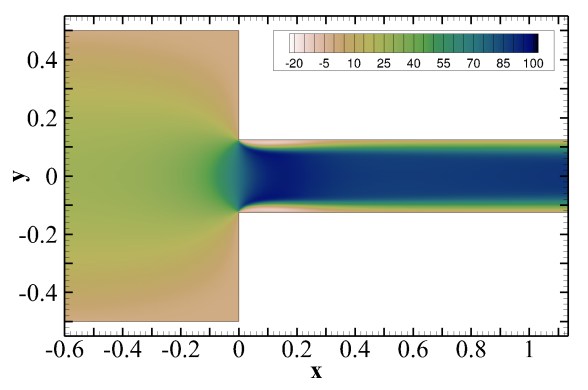

(a) $R e=1000$.

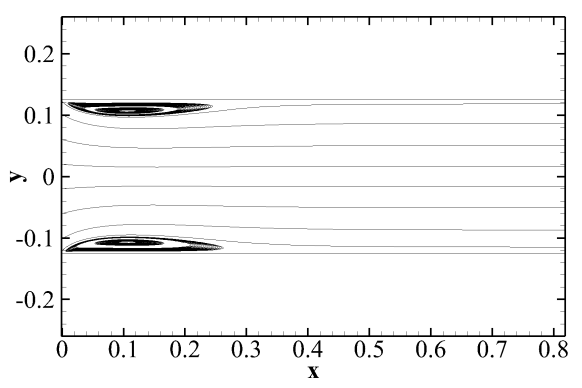

(c) $R e=1000$, zoomed region.

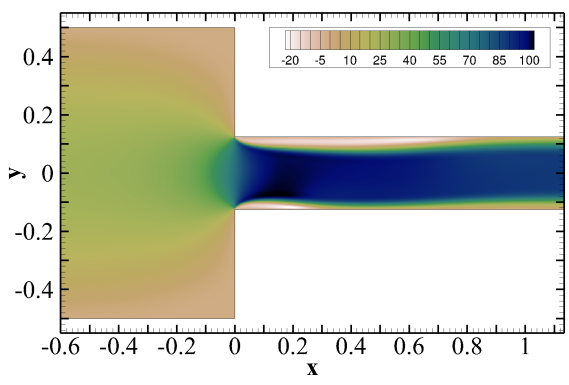

(b) $R e=2000$.

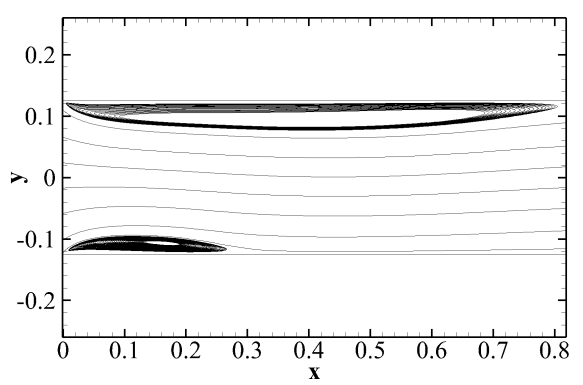

(d) $R e=2000$, zoomed region.

Figure 3: Horizontal velocity component $U$ (a,b) and streamlines (c,d) for $R e=1000$ and $R e=2000$, showing symmetric and asymmetric recirculation bubbles, respectively. Contraction ratio is $C=4$ in all cases.

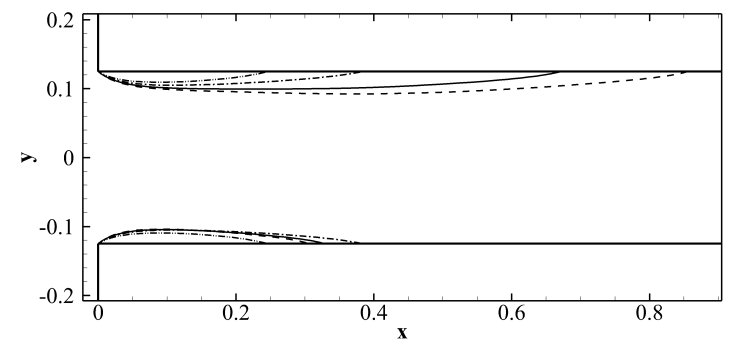

Figure 4: Recirculation bubbles for contraction ratio $C=4$ at $R e=800$ (dash dot dot), $R e=1200$ (dash dot), $R e=1600$ (solid) and $R e=2000$ (dash).

\section{OPTIMISATION}

In this section, we first detail the methodology for shape optimisation. In particular, we introduce the optimisation procedure based on a genetic algorithm, the parametrisation of the geometry (and associated mesh deformation), the mode tracking method that enables the identification of the most unstable asymmetric eigenmodes and the surrogate model enabling an efficient optimisation.

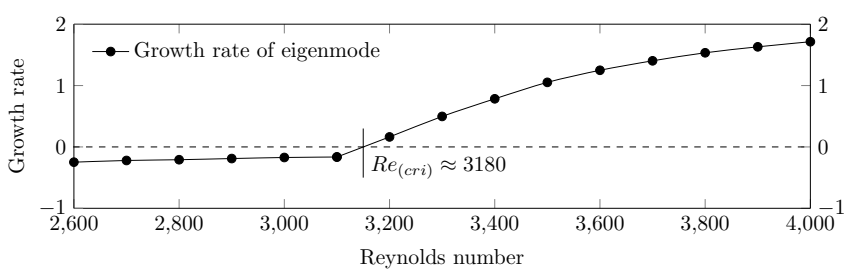

(a) $C=2$.

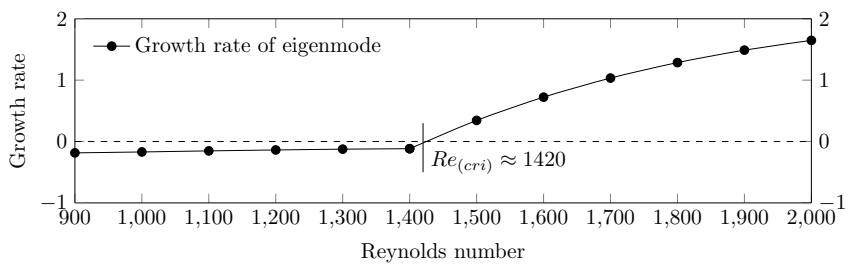

(b) $C=4$.

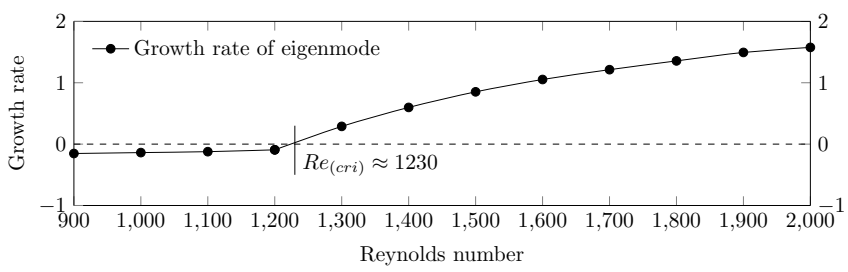

(c) $C=8$.

Figure 5: Growth rates (real part of eigenvalues) against Reynolds numbers, for $C=2,4$ and 8 . 


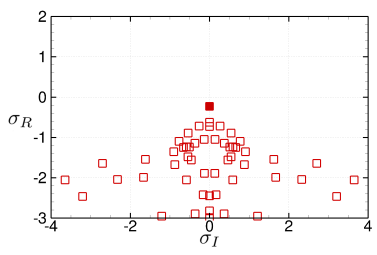

(a) $R e=1000$.

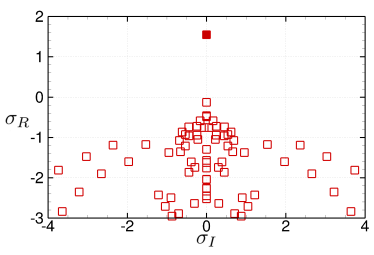

(b) $R e=2000$.
Figure 6: Eigenvalue spectra for $R e=1000$ and 2000 for contraction ratio $C=4$. The least stable mode is marked with a filled square.

\section{A. Methodology}

Bearing in mind that real part of the eigenvalue $\left(\sigma_{R}\right)$ stands for the growth rate of eigenmodes, the optimisation includes the minimisation of a cost function, which is defined through the real part of the eigenvalue responsible for the onset if asymmetry $\left.\sigma_{R}\right|_{\text {assym. }}$. This eigenvalue is a function of the parameters $\boldsymbol{p}$ defining the channel geometry. Based on this assumption, the optimisation problem may be stated as:

$$
\begin{aligned}
& \left.\min \sigma_{R}\right|_{\text {assym }}(\boldsymbol{p}) \\
& \text { subject to: } h_{i}(\boldsymbol{p})=0, \text { for } i=1,2 \\
& \text { min }\left(\left.\sigma_{R}\right|_{\text {buf }}\left(\boldsymbol{p}_{\mathbf{1}}\right), \quad C_{d}\left(\boldsymbol{p}_{\mathbf{2}}\right)\right) \\
& \text { subject to }: h_{i}(\boldsymbol{p})=0, \text { for } i=1,2 \\
& \text { min } C_{d}\left(\boldsymbol{p}_{\mathbf{2}}\right) \\
& \text { subject to: } h_{i}(\boldsymbol{p})=0, \text { for } i=1
\end{aligned}
$$

in which, $\boldsymbol{p}$ is the set of selected parameters defining the geometry (defined in the next section). The flows in the new optimised shapes need to fulfil at all times the NavierStokes Eq. (1) and the eigenvalue problem Eq. (3), both defined in previous section III. More specifically, these constraints may be stated by imposing $h_{1}=\mathscr{M} \frac{\partial \boldsymbol{q}}{\partial t}-\mathscr{F}(\boldsymbol{q})$ and $h_{2}=\mathscr{J}(\overline{\boldsymbol{q}}) \hat{\boldsymbol{q}}-\boldsymbol{\sigma} \mathscr{M} \hat{\boldsymbol{q}}$ in the minimisation problem Eq. (9).

Based on the optimisation problem, the optimisation loop requires a surrogate model, a geometry parametrisation, a mesh deformation, a base flow simulation, a stability analysis and a mode tracking. The main loop is started by building the initial surrogate model. Then, followed by the loop of optimisation and surrogate model updating. The flowchart of the optimisation is shown in Fig. 9. The algorithm requires the following steps detailed in the figure. For each iteration of (d), part (c) is executed. Each execution of (c) requires one call to (b) and (a), and every time that (b) is executed, (a) is also executed.

\section{Genetic Algorithm}

Genetic algorithm emulate the process of natural selection and genetic mechanism in Darwin's theory of evolution, and
Table VII: Parameters selected for the Genetic Algorithm.

\begin{tabular}{llc}
\hline \hline Name & Representation & Value \\
\hline$N_{G}$ & Number of maximum generations & 500 \\
$N_{p}$ op & Number of population & 100 \\
$N_{g}$ & Number of genes & 3 \\
$P_{x}$ & Possibility of crossover & 0.6 \\
$P_{m}$ & Possibility of mutation & 0.6 \\
\hline \hline
\end{tabular}

seek the optimal solution through a natural genetic process ${ }^{54}$. The genetic algorithm starts with an initial population (a set of potential solutions), which consists of a number of individuals with combinations of different genes. A gene is a member in a parameter array of an individual. By calling a cost function, the fitness of each individual is evaluated, and the best individuals are selected. After crossover and mutation of genes, new generations with higher fitness are obtained. After a certain amount of iterations, an optimal solution is reached. The procedure of a typical genetic algorithm is shown in Fig. 10. In the algorithm, the number of maximum generations, the number of populations, the number of genes, the possibility of crossover and the possibility of mutations, need to be specified. The parameters retained for our optimisation are summarised in Table VII. Further details on genetic algorithms may be found in Ref. 55 .

\section{Parametrisation and Mesh Deformation}

The geometry is parametrised using super-elliptic curves of the form:

$$
\left|\frac{x}{a}\right|^{1 / p}+\left|\frac{y}{b}\right|^{1 / p}=1
$$

In our implementation, we set $a=b=1$, as it proves favourable that the major and minor axis of the super-ellipse are equal, to reduce the number of the geometry parameters and computational cost. With higher degree $p$, the curvature radius becomes smaller and enables the definition of a straight angle as $p$ tends to zero or infinity, as shown in the Fig. 11. This parametrisation provides a continuous curvature at the intersection point between the parametrised part and the remaining geometry, allowing smooth transitions between different boundary sections. This last property helps to deform the computational mesh and alleviates the apparition of distorted elements.

As shown in Fig. 7 and discussed in section III B 1, the mode responsible for the onset of asymmetries is located near the salient corner and the tip corner. Consequently, we parametrise the geometry such that the optimisation algorithm can reshape the geometry near these regions. The top corner and salient corner (at the contraction stage) are parametrised as two distinct super-elliptic curves, with exponents in Eq. (10) being $p_{1}$ for the top and $p_{2}$ for the salient corner. As shown in Fig. 11, the degree of the super-elliptic curve controls the curvature of the corners. An additional parameter is included to control the tangential direction of the contraction 


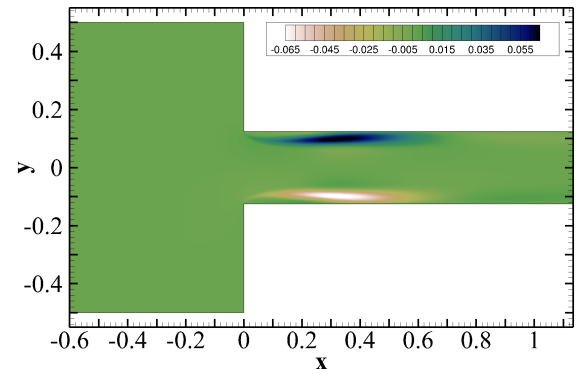

(a) Direct eigenmode, $R e=1000$.

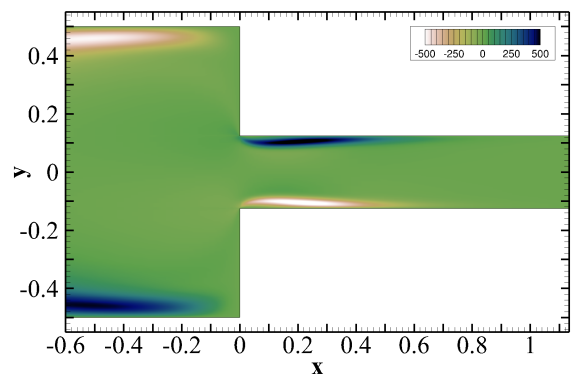

(c) Adjoint eigenmode, $R e=1000$.

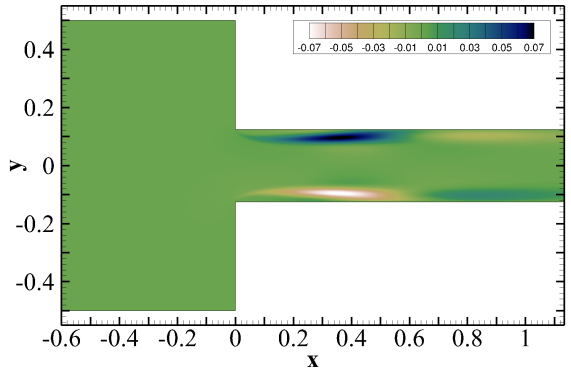

(b) Direct eigenmode, $R e=2000$.

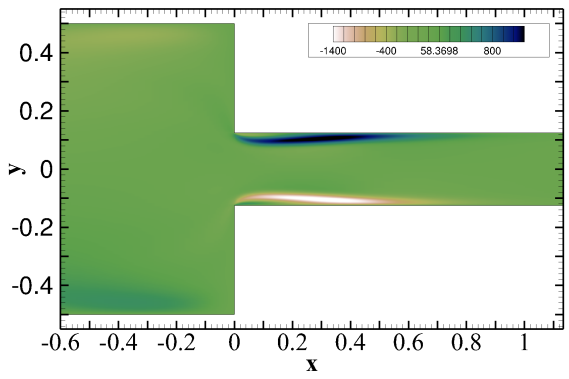

(d) Adjoint eigenmode, $R e=2000$.

Figure 7: Direct (a,b) and adjoint $(\mathrm{c}, \mathrm{d})$ eigenmodes for $R e=1000$ and $R e=2000$, for contraction ratio $C=4$. All show horizontal velocity component $u$.

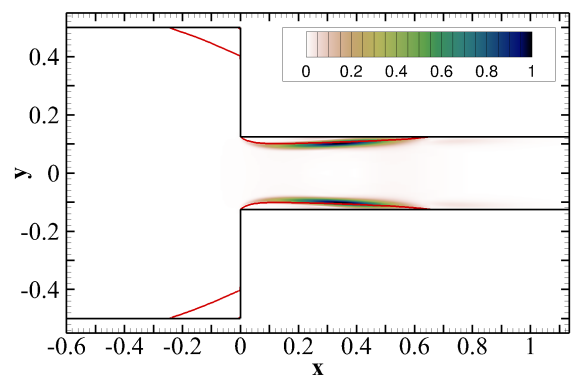

(a) Spatial map of structural sensitivity.

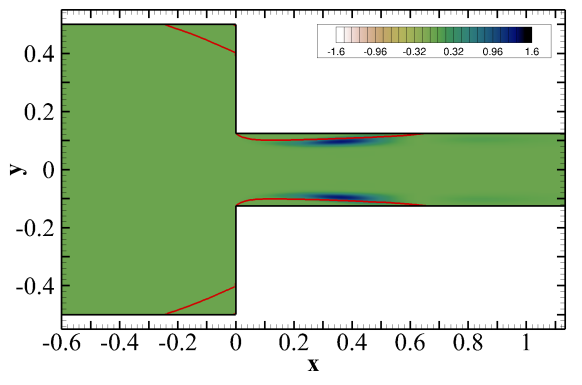

(b) Production of the perturbation energy.

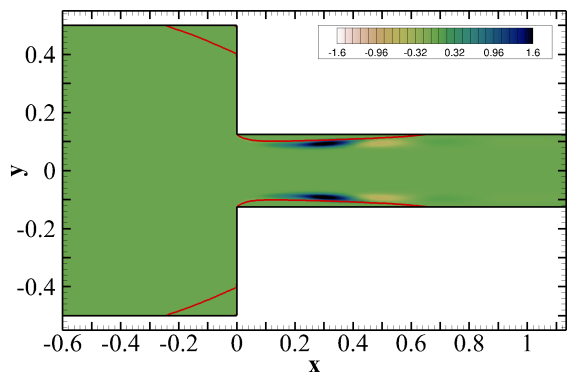

(c) Lift-up perturbation: $u v d U / d y$.

Figure 8: Structural sensitivity map, Production of kinetic energy and lift-up kinetic energy perturbation, at $R e=2000$ for contraction ratio $C=4$. Red lines indicate the edge of recirculation regions. 


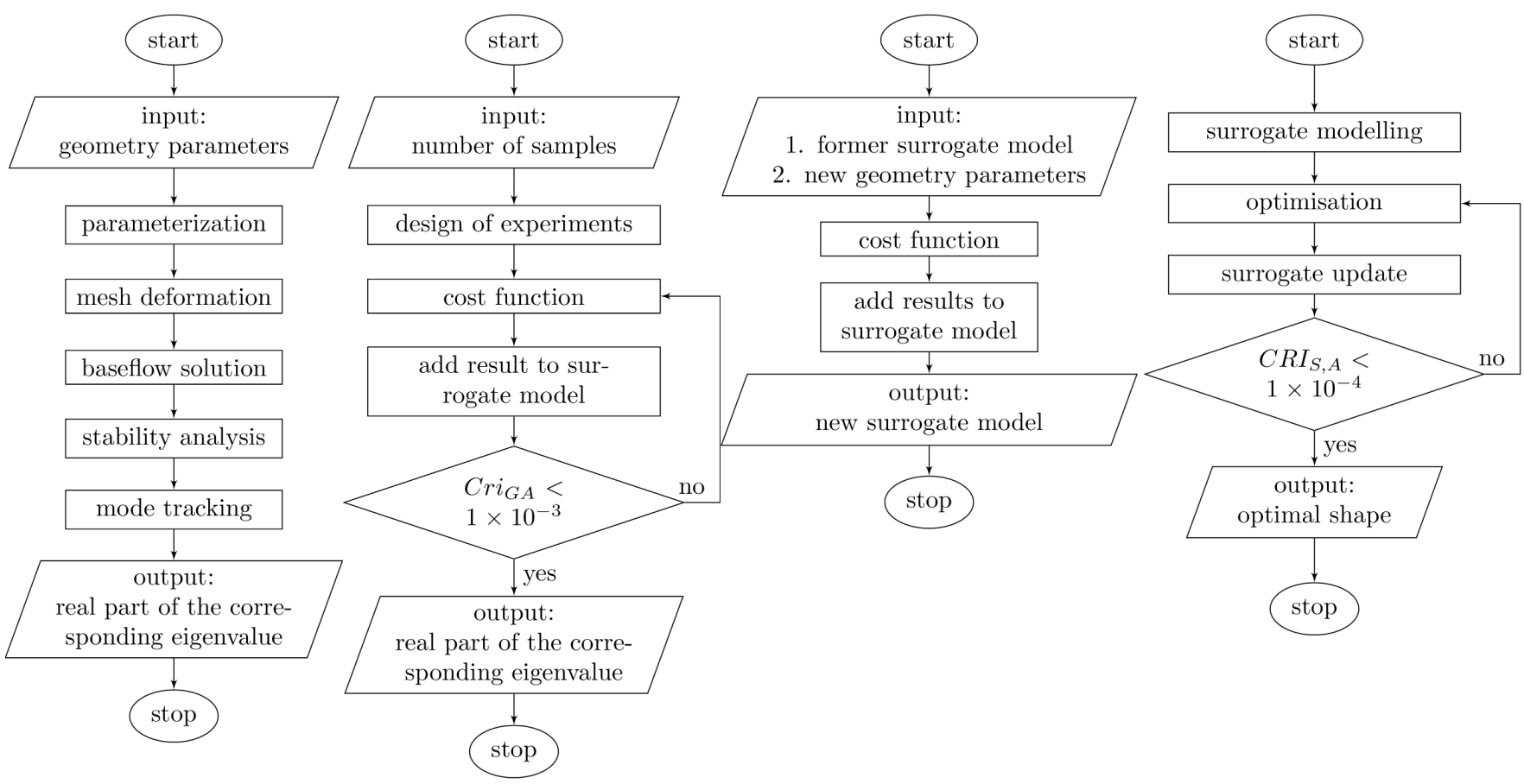
(a) Cost function.
(b) Surrogate model.
(c) Surrogate update.
(d) Optimisation.

Figure 9: The optimisation loop.

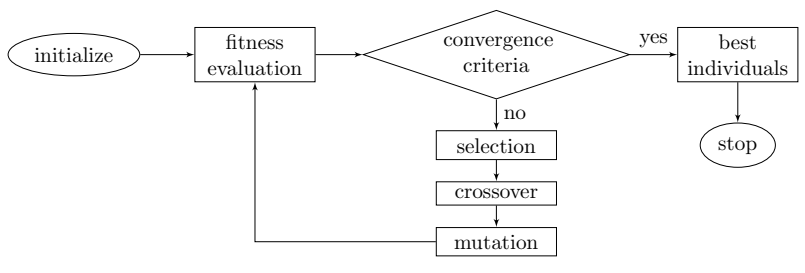

Figure 10: Flow chart of a simple genetic algorithm.

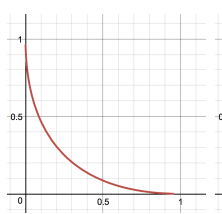

(a)

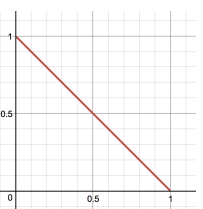

(b)

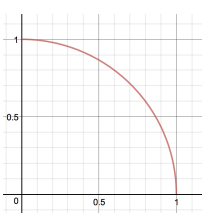

(c)

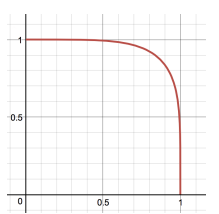

(d)
$(1.0,1.0,0.5)$.
$(1.0,1.0,1.0)$.
$(1.0,1.0,2.0)$.
$(1.0,1.0,5.0)$

Figure 11: Super-elliptic curves with different degrees, with parameters $(a, b, 1 / p)$.

stage with respect to the vertical direction. In summary, the set of parameters for optimisation $\boldsymbol{p}=\left(p_{1}, p_{2}, p_{3}\right)$, see Eq. (9), include a parametrisation of the two corners through $p_{1}$, $p_{2}$. Additionally, $p_{3}$ governs the angle (in degrees) of the contraction stage with respect to vertical direction. These parameters are sketched in Fig. 12 and their ranges (or complete design space) are summarised in Table VIII. Let us note that the

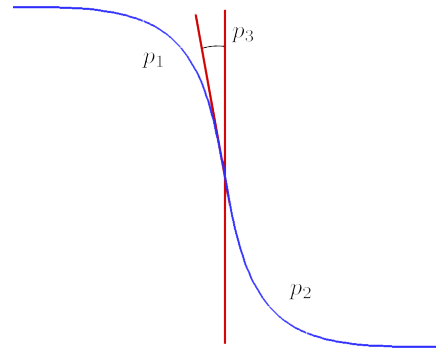

Figure 12: Geometry parametrisation using the parameters $\boldsymbol{p}=\left(p_{1}, p_{2}, p_{3}\right)$ at the contraction stage.

Table VIII: Range for the geometry parameters (or design space): $\boldsymbol{p}=\left(p_{1}, p_{2}, p_{3}\right)$.

\begin{tabular}{cccc}
\hline \hline Parameter & Lower bound & Upper bound & Original \\
\hline$p_{1}$ & 0.0 & 1.0 & 0.0 \\
$p_{2}$ & 0.0 & 1.0 & 0.0 \\
$p_{3}$ & $-2.5^{\circ}$ & $2.5^{\circ}$ & 0.0 \\
\hline \hline
\end{tabular}

selected range of parameters only allow small deformations, whilst retaining the overall shape of the contraction channel.

For completeness, we show examples of the parametrisation in Fig. 13, the the minimum and maximum values for each parameter and the resulting shapes are illustrated.

Since the shape optimisation will deform the geometry, it is important to control the mesh deformation to avoid unusable meshes (e.g. with negative or distorted elements). To con- 


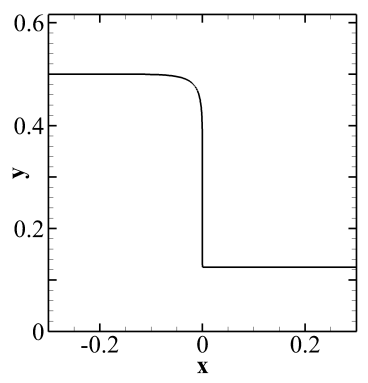

(a) $\left(1.0,0.0,0.0^{\circ}\right)$.

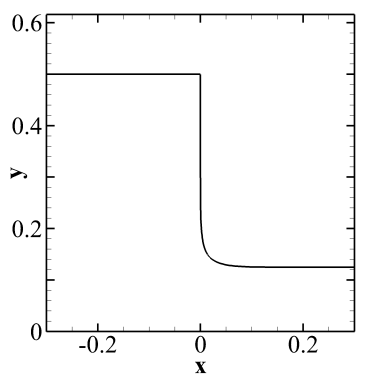

(b) $\left(0.0,1.0,0.0^{\circ}\right)$.

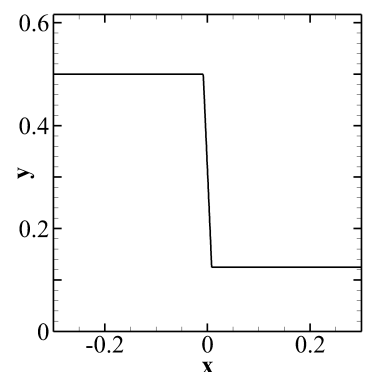

(c) $\left(0.0,0.0,2.5^{\circ}\right)$.

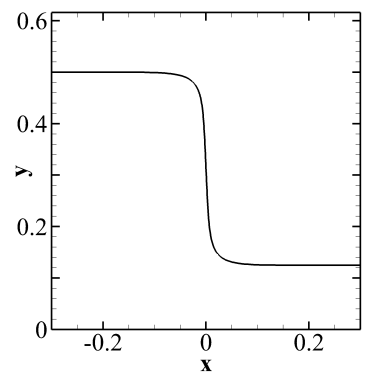

(d) $\left(1.0,1.0,2.5^{\circ}\right)$.

Figure 13: Examples of geometries using the geometry parametrisation $\boldsymbol{p}=\left(p_{1}, p_{2}, p_{3}\right)$.

trol the mesh deformation, a Free-form deformation (FFD) method is developed based on Ref. 56. The FFD acts on the two-dimensional boundary mesh deforming the inner grid, whilst maintaining the quality of the mesh. Some examples of the mesh deformation are given with different geometry parameters in Fig. 14, where it can be seen that the mesh is fine enough even in the modified geometries.

\section{Surrogate Modelling}

Surrogate models are often adopted in the process of optimisation with complex cost functions, to reduce calls of cost function evaluation, reducing the impact of uncertainty in evaluation and for easier parallel off-line evaluation, which is particularly important when considering expensive evaluations such as when considering base flow and stability analysis. Surrogate models can be divided into two categories by whether they require gradient information or not. The ones without gradient information are more commonly utilised in global optimisation together with algorithms that do not require gradients of the cost functions, like genetic algorithm. There are a number of gradient-free surrogate models in the literature, including RSM (polynomial Response Surface Model), Kriging or RBFs (Radial Basis Functions) ${ }^{43}$. Among the surrogate models, the regression models like RSM are well suited for local optimisation problems with relatively simpler design space, while interpolation models such as Kriging and RBF can be used for highly-nonlinear multi-model functions, and thus well suited for global optimisation problems with complicated design space ${ }^{57}$. Alongside Kriging, RBF (Radial Basis Function) interpolation is a popular method for constructing surrogates suitable for highly non-linear data using irregular distributions of sample points, which can be adapted to the function being modelled. RBFs have seen increasing use for aerospace applications requiring an interpolation or approximation method, including mesh motion, fluidstructure interaction, and domain element parametrisation, in addition to aircraft data interpolation, owing to the meshless and general nature of the formulation, and tolerable properties of the interpolation kernel ${ }^{58}$.

A Radial Basis Function (RBF) method is a linear com- bination of a series of basis functions, whose argument is the Euclidean distance between the interpolation point $\boldsymbol{p}=$ $\left(p_{1}, p_{2}, p_{3}\right)$ and all the other points in the known data set. The model has the form:

$$
\sigma_{R}(\boldsymbol{p})=\sum_{i=1}^{N} w_{i} \phi\left\|\boldsymbol{p}-\boldsymbol{p}_{i}\right\|
$$

where $\boldsymbol{p}$ is the interpolation point, $\phi$ is the chosen basis function, $w_{i}$ is the weight coefficient for each basis function, and $\|\bullet\|$ represents the Euclidean norm. Any function $\phi$ that satisfies the property $\phi(\boldsymbol{p})=\phi(\|\boldsymbol{p}\|)$ is a radial function, and can be used as a basis function in RBFs. In this work, we select as many RBFs as the number of sampling points for the construction of the surrogate model (see next sections). As applied to the current investigation, $\sigma_{R}(\boldsymbol{p})$ is the growth rate (real part of the eigenvalue) governed by the geometry parameter array $\boldsymbol{p}=\left(p_{1}, p_{2}, p_{3}\right)$.

\section{Design of Experiments}

Design of Experiments enable building surrogate models with minimal sampling of the cost function (in this case, base flow solution and stability analysis ${ }^{59}$. The most commonly employed experimental design methods are currently full factorial design, orthogonal design, uniform design, Latin hypercube sampling, central composite design, etc. Considering both the computational cost of the objective function evaluation and the construction of the efficiency and accuracy of the surrogate model, orthogonal design is adopted in the present work. Orthogonal experimental design, which is a partial design of full factorial design, is an efficient compromise between computational cost and accuracy of the surrogate model.

Instead of having $L^{N}$ sampling points in a full factorial design, an orthogonal design has $L^{N-1}$ sampling points, having $L$ levels for each parameter. In this work, there are $N=3$ parameters $\left(p_{1}, p_{2}, p_{3}\right)$, each of which takes $L=5$ different levels (or degrees) making a $L^{N-1}=5^{2}$ orthogonal design experiment, with 25 sampling points. 

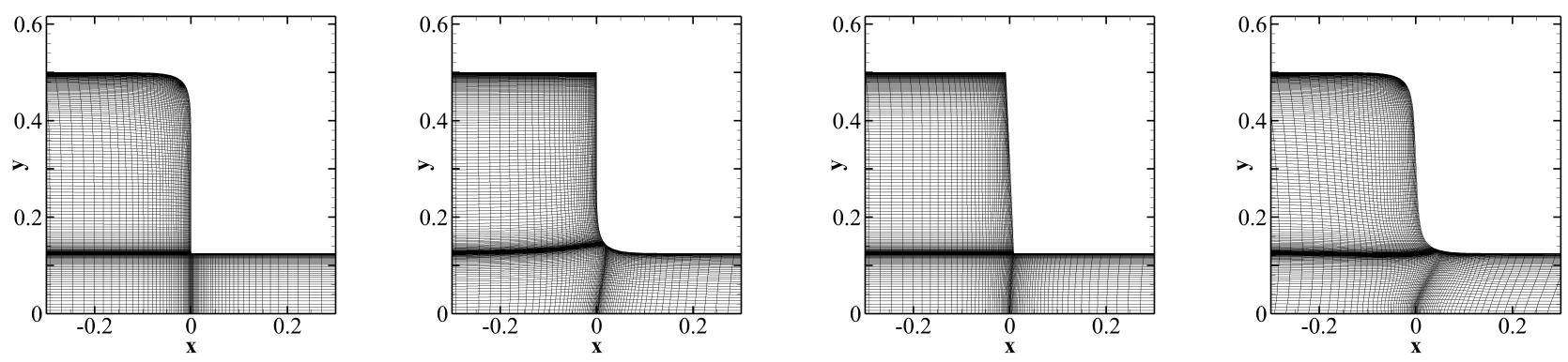

Figure 14: Examples of mesh deformation for the geometries depicted in Fig. 13.

\section{Mode Tracking}

To include the stability analysis in the optimisation loop, it is necessary to avoid manual identification of the eigenmodes obtained with new channel shape (i.e. identify the eigenmode responsible for the onset of asymmetry). A Modal Assurance Criterion (MAC) ${ }^{60}$ is incorporated into the optimisation loop. The MAC method is an efficient way to track the modes by comparing sets of eigenmodes. To rank the similarity between eigenmodes, a MAC index is defined for a pair of eigenmodes $\hat{\boldsymbol{q}}_{i}$ and $\hat{\boldsymbol{q}}_{j}$ :

$$
\operatorname{MAC}\left(\hat{\boldsymbol{q}}_{i}, \hat{\boldsymbol{q}}_{j}\right)=\left(\frac{\left|\hat{\boldsymbol{q}}_{i} \cdot \hat{\boldsymbol{q}}_{j}\right|}{\left\|\hat{\boldsymbol{q}}_{i}\right\|\left\|\hat{\boldsymbol{q}}_{j}\right\|}\right)^{2} .
$$

The MAC index approaches 1 when the pair of eigenmodes are linearly dependent (or identical), and is zero for orthogonal eigenmodes (not similar shapes).

The algorithm is more reliable when using eigenmodes computed on the same mesh. In this investigation, we modify the algorithm to allow comparison of eigenmodes in different meshes. The resulting method requires a few steps. First, all the the eigenmodes of interest are fed to the program. Then, the program process a first match to find the eigenmodes that have similar eigenvalues. After eliminating the ones with large eigenvalues differences, the program interpolates the eigenmode into the same mesh to generate a new set of comparable modes. Then, these are matched using the MAC method, which works well when using the same mesh.

\section{B. Optimisation Results and Discussion}

In this section, we discuss the result obtained through shape optimisation of the contraction channel, with the aim of increasing the critical Reynolds number. To obtain results that may be compared to optimisations based on stability and sensitivity analyses (e.g. Lashgari et al. Lashgari et al. ${ }^{17}$ for sensitivities to blowing/suction), we only allow small shape deformations. We select one unstable Reynolds number for each contraction ratio $\left(R e_{o p t}\right.$, slightly higher that the critical Reynolds number, see Fig. 2), and optimise. We choose
Table IX: Total number of iterations and computational time taken for the optimisation.

\begin{tabular}{ccc}
\hline \hline$C$ & $N_{c}$ & $T_{c}($ core $\times$ hour $)$ \\
\hline 2 & 16 & 337 \\
4 & 19 & 427 \\
8 & 17 & 439 \\
\hline \hline
\end{tabular}

$R e_{o p t}=3200$ for $C=2, R e_{o p t}=1500$ for $C=4$ and $R e_{o p t}=$ 1300 for $C=8$.

In this optimisation investigation, we select two criteria to check the convergence of the optimisation procedure, $C R I_{S, A}$ and $\mathrm{Cri}_{G A}$, as shown in Fig. 9. First, in the procedure of surrogate model (shown in Fig. 9b), we check the relative change of the cost function value obtained from the surrogate model of the current generation compared to the last generation, $\mathrm{Cri}_{G A}$. Second, in the optimisation loop (shown in Fig. 9d), we monitor the error of the optimal obtained by GA from the surrogate and compare it to the value of cost function from the actual simulation at this given point, $C R I_{S, A}$. We choose $C R I_{S, A}<1 \times 10^{-4}$ and $\mathrm{Cri}_{G A}<1 \times 10^{-3}$ as the convergence criteria for the present optimisation investigation.

In Figs. 15, we show the convergence process of the optimisation on the contraction channels with three contraction ratios. It is noticeable that the error of the surrogate model is un-neglectable in the beginning. However, with the dynamical updating scheme, the error decreases as the optimisation progresses. In less than 20 iterations, the error of the surrogate model, which is the criterion we choose for the optimisation $\left(C R I_{S, A}\right)$, becomes lower than $1 \times 10^{-4}$, and the optimisation is terminated. It is also shown that the grow rates of the eigenmodes causing the bifurcation are greatly reduced to below zero. Total number of iterations taken to converge $\left(N_{c}\right)$ and computing hours $\left(T_{c}\right)$ are shown in Table IX for the three contraction ratios.

When the optimal shape is obtained, it is important to test the optimal shape against some random suboptimal shapes, as previous study by Tammisola ${ }^{61}$. After the optimisation is converged, we compare the optimised geometries to the geometries consisting the sample group of the surrogate model and the geometries updated to the surrogate model. All these geometries show less stable flow comparing to the optimal shape 


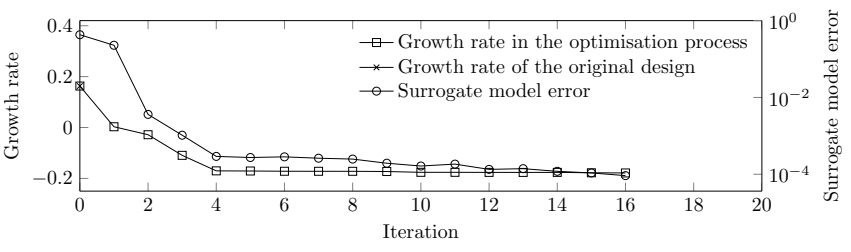

(a) $C=2$.

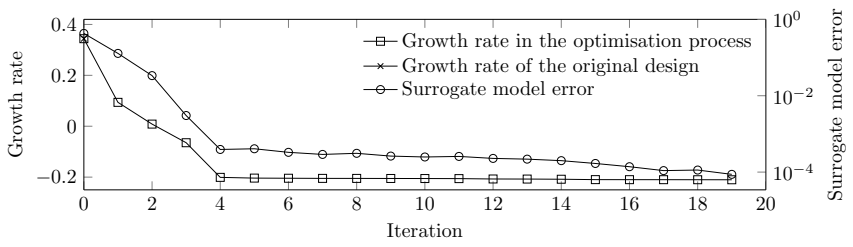

(b) $C=4$.

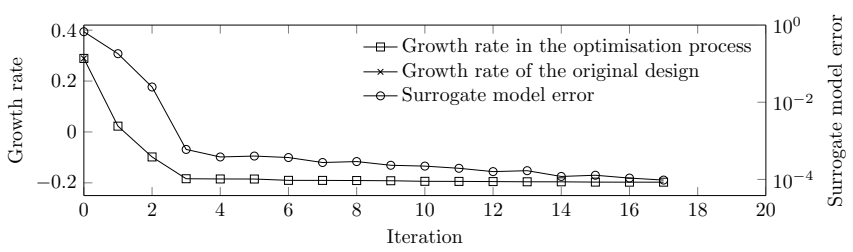

(c) $C=8$.

Figure 15: Convergence process of the optimisation.

obtained after the optimisation is converged.

The shapes resulting from the optimisation are depicted in Figs. 16, 17 and 18. In these figures, the original shapes have also been included for comparison. In all cases, we observe that the tip and salient corners have been rounded and that the contraction stages (vertical walls) have been tilted.

We can see in the eigenvalue spectra, shown in Figs. 16b, $17 \mathrm{~b}$ and $18 \mathrm{~b}$ in comparison with Figs. 16a, 17a and 18a that the growth rates of the most unstable asymmetric modes for each contraction ratio are reduced and below zero in the optimised shapes. It is clear that the optimisation has stabilised the mode responsible for asymmetries but also that the shape deformation has not destabilised other modes. The corresponding eigenmodes are shown in Figs. 16d, 17d and 18d in comparison with Figs. 16c, 17c and 18c. The modes in the optimised geometries are thinner and have been relocated from behind the tip corners to around the corner. These new modes lie along the boundaries following the the curved corner. It is noticeable how small changes in the geometry modify substantially the eigenmodes.

Additionally, we show the base flows for the original and optimised geometry configurations. Results for the optimised shapes are shown in Figs. 16f, $17 \mathrm{f}$ and 18f, in comparison with the original results shown in Figs. 16e, 17e and 18e. After the optimisation, we observe a significant reduction of the velocity gradients at the contraction. Furthermore, the flows are all symmetric and the sizes of the recirculation bubbles are either greatly reduced $(C=2)$ or even completely eliminated $(C=4$ and $C=8)$.

Sensitivity analyses are compared for the original and optimised configurations (and for the three contraction ratios).
The structural sensitivity maps for the optimised configurations are shown in Figs. 16h, 17h and 18h, which can be compared to the sensitivities of the original configurations, Figs. $16 \mathrm{~g}, 17 \mathrm{~g}$ and $18 \mathrm{~g}$. For all three contraction ratios, the regions with high sensitivity have been relocated from near the tip corner bubbles to the salient corner bubbles, which shows the beneficial effect of the optimisation in stabilising the mode responsible for asymmetries. Additionally, the liftup perturbation kinetic energy $(u v d U / d y)$ has almost disappeared illustrating how the new shapes minimise the lift-up mechanisms resulting in more stable configurations. It is important to note that structural sensitivity and lift-up term show that the physical mechanism behind the pitch fork bifurcation has been changed by very small modification in the geometry.

We now analyse the sensitivity of the shape parameters selected for optimisation with respect to the growth rate. Fig. 19 shows the influence of the parameters in the growth rates of the asymmetric modes, and provides valuable information on the influence of each parameter in the flow stability. We can see for three different contraction ratios that the angle of the contraction stage (vertical walls) affects the growth rate the most whilst the degree of the super elliptic curve at the salient corner affects the least. This parameter-sensitivity analysis suggests that the optimised configurations are optimal within the design space. It is important to note that all optimised configurations reach the edge of the design space, which means that if the design space is enlarged, the optimised configurations may go beyond the current design space. However, we have shown that this design space, which was narrowly restricted to avoid large deformations, is sufficient to enhance flow stability and avoid unsymmetrical flows.

Then, to quantify the increase in critical Reynolds numbers, we include a bifurcation study or the optimised geometries and the three contraction ratios $C=2,4$ and 8 . The size of the recirculation region at the critical Reynolds number has decreased for $C=2$ and 4 . The reattachment lengths against Reynolds numbers of the new shapes of $C=2$ and 4 are shown in Fig. 20.

More interestingly, we can quantify the increase in the critical Reynolds numbers $\left(R e_{c r i}\right)$. For the optimised shapes, these values are $R e_{c r i}=24800$ and $R e_{c r i}=27900$ for $C=2$ and 4 respectively. In comparison with the original geometry, in which the critical values were $R e_{c r i}=3150$ and $R e_{c r i}=1420$, the critical Reynolds numbers are vastly increased by 7.9 and 20.9 times for contraction ratio $C=2$ and 4 .

Additionally, as for contraction ratio $C=8$, note that there are no signs of bubbles near the tip corners for all the Reynolds numbers tested, which is as high as $4 \times 10^{6}$, shown in Fig. 21 . Thus, the critical Reynolds number is believed not to exist within the range of selected Reynolds numbers.

These results prove that the Reynolds number, at which the bifurcation occur, can be greatly increased through a shape optimisation that includes stability analysis information as the target functional and leads to very small shape deformations. 


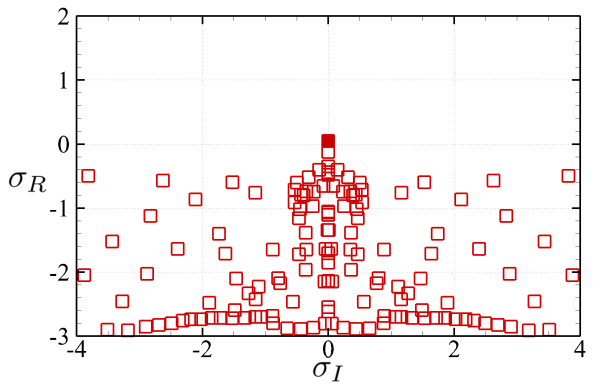

(a) Original eigenvalue spectrum.

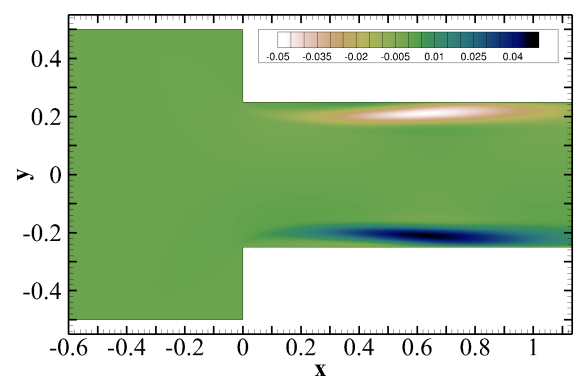

(c) Original eigenmode (horizontal velocity).

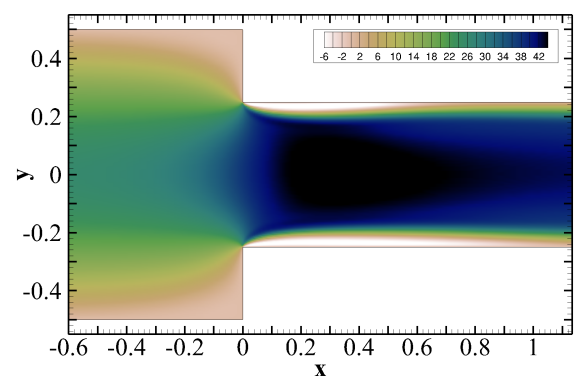

(e) Original base flow (horizontal velocity).

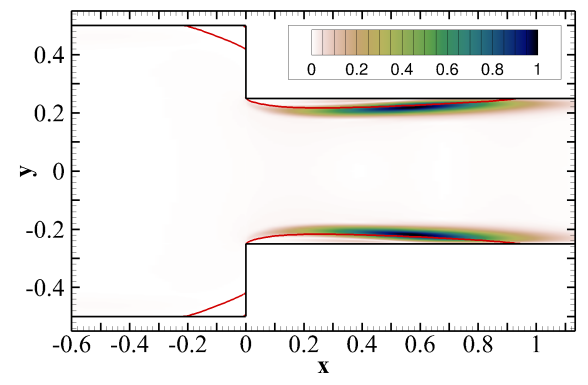

(g) Original structural sensitivity.

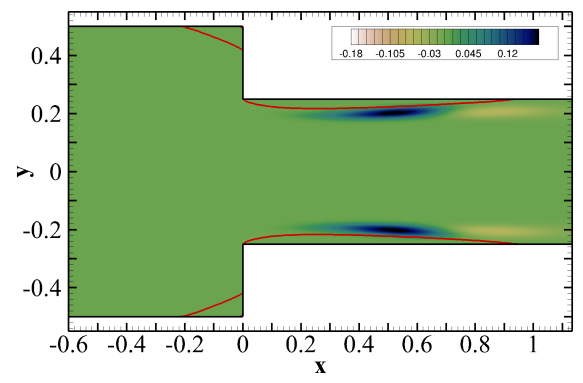

(i) Original lift-up perturbation: $u v d U / d y$.

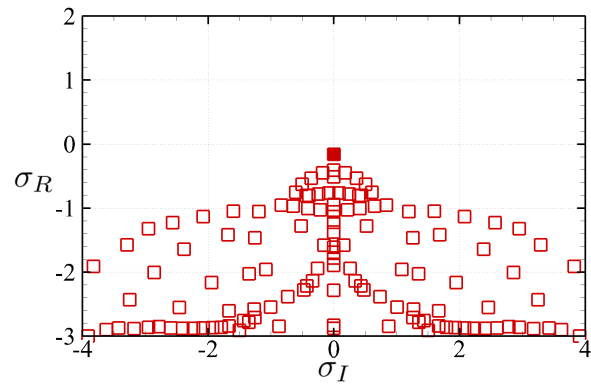

(b) Optimised eigenvalue spectrum.

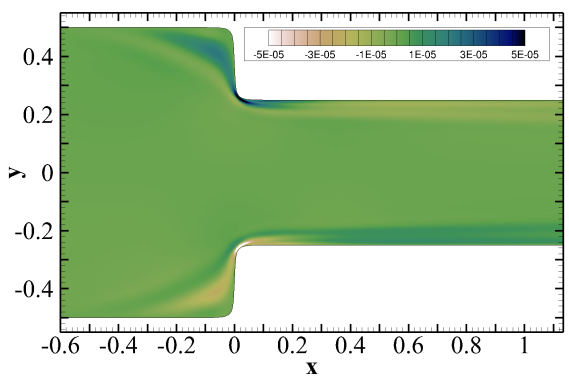

(d) Optimal eigenmodes (horizontal velocity).

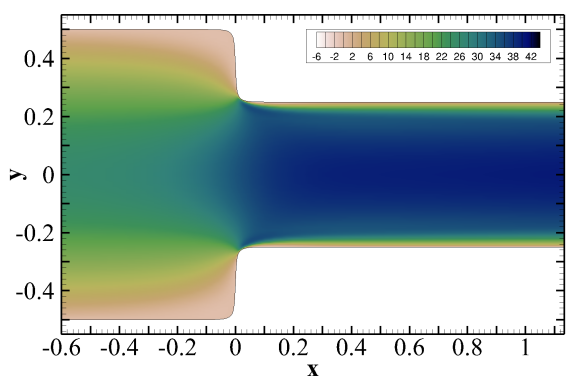

(f) Optimised base flow (horizontal velocity).

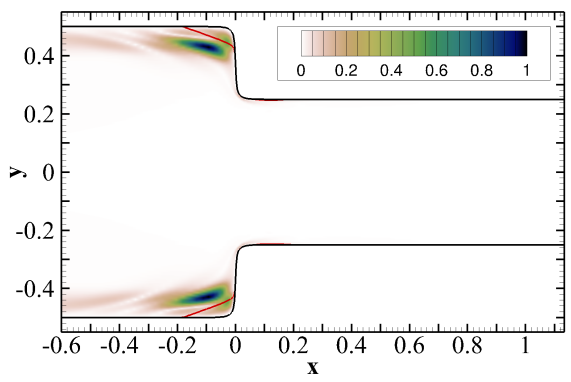

(h) Optimised structural sensitivity.

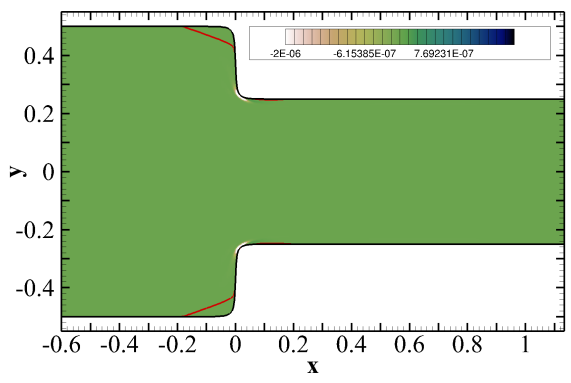

(j) Optimised lift-up perturbation: $u v d U / d y$.

Figure 16: Original and optimised geometries for contraction ratio $C=2$ : eigenvalue spectra, eigenmodes, base flows, structural sensitivities and lift-up perturbation kinetic energy terms. Red lines indicate the edge of recirculation regions. 


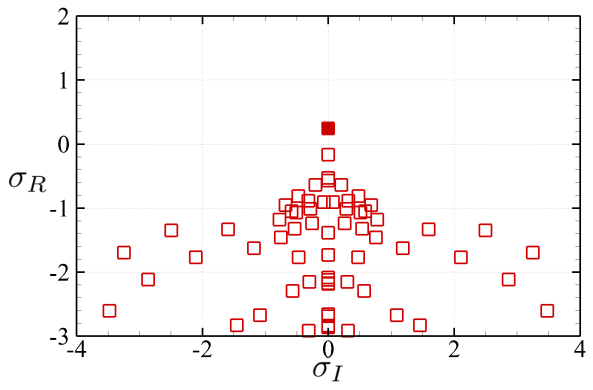

(a) Original eigenvalue spectrum.

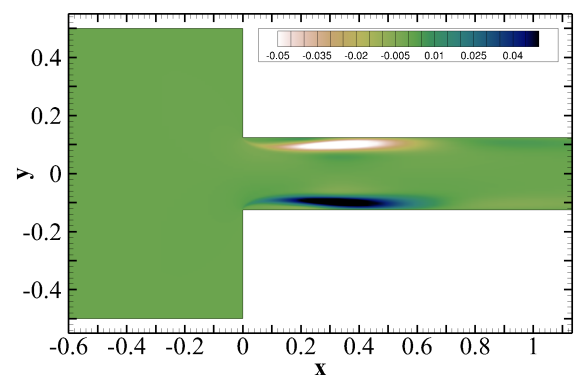

(c) Original eigenmode (horizontal velocity).

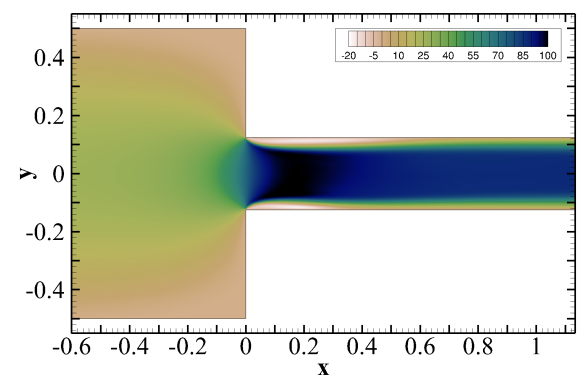

(e) Original base flow (horizontal velocity).

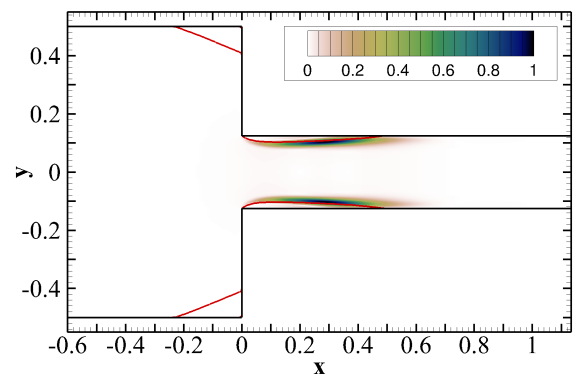

(g) Original structural sensitivity.

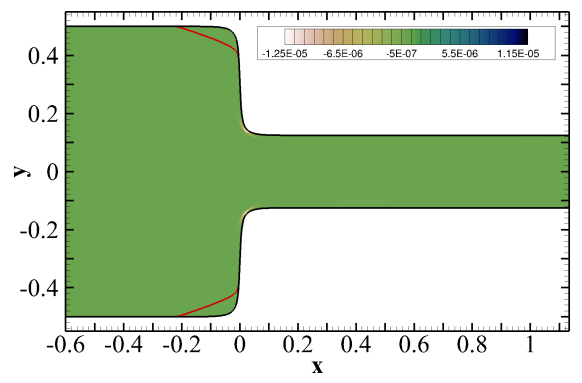

(i) Original lift-up perturbation: $u v d U / d y$.

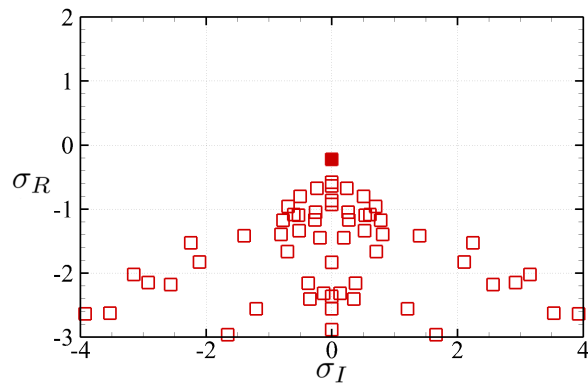

(b) Optimised eigenvalue spectrum.

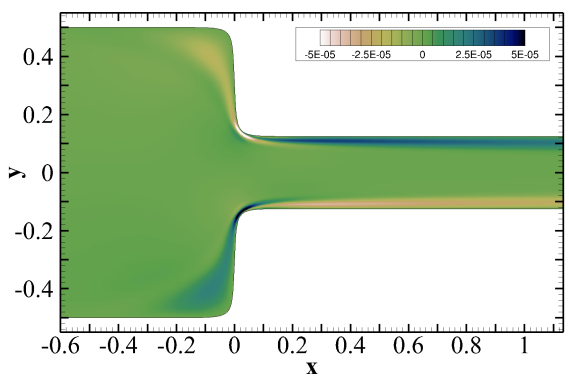

(d) Optimised eigenmodes (horizontal velocity).

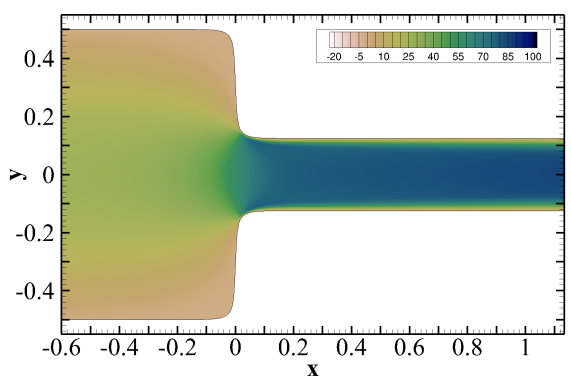

(f) Optimised base flow (horizontal velocity).

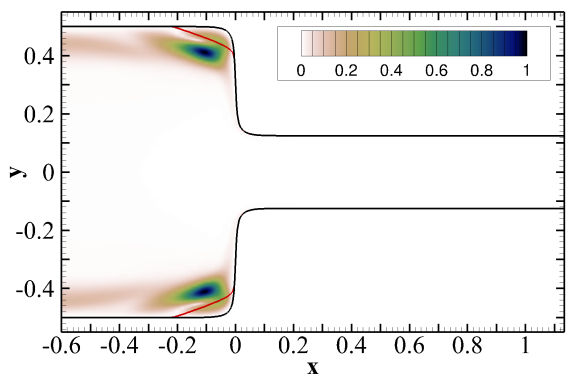

(h) Optimised structural sensitivity.

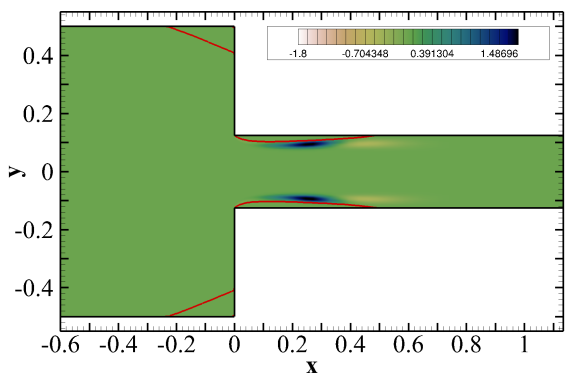

(j) Optimised lift-up perturbation: $u v d U / d y$.

Figure 17: Original and optimised geometries for contraction ratio $C=4$ : eigenvalue spectra, eigenmodes, base flows, structural sensitivities and lift-up perturbation kinetic energy terms. Red lines indicate the edge of recirculation regions. 


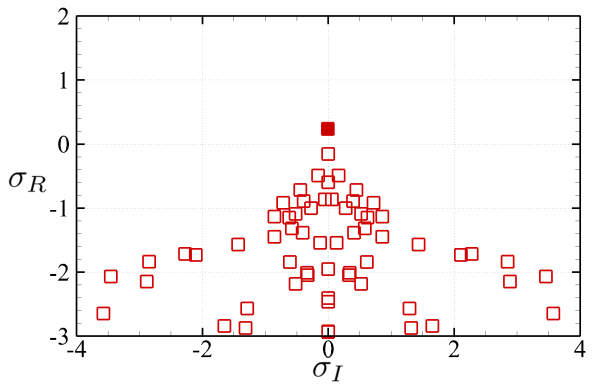

(a) Original eigenvalue spectrum.

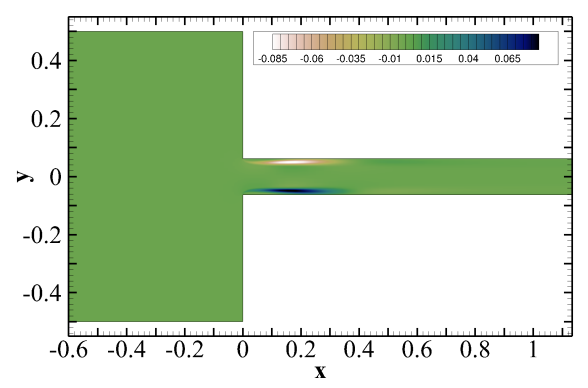

(c) Original eigenmode (horizontal velocity).

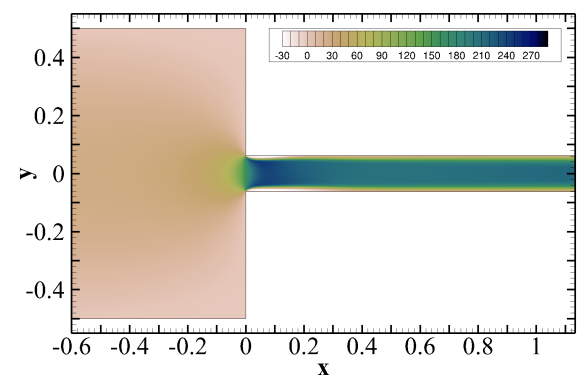

(e) Original base flow (horizontal velocity).

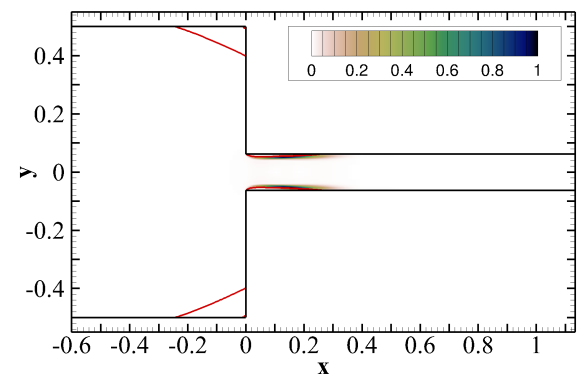

(g) Original structural sensitivity.

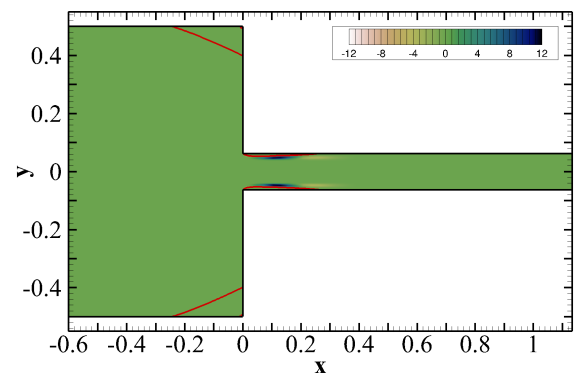

(i) Original lift-up perturbation: $u v d U / d y$.

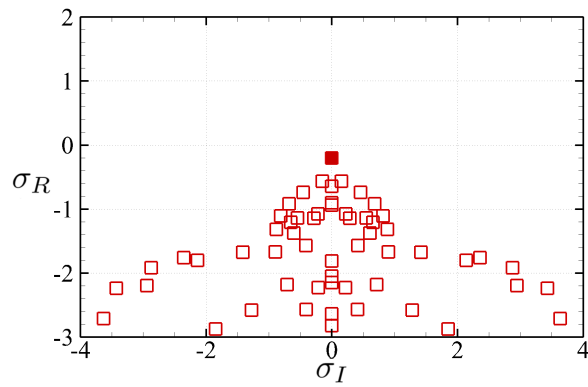

(b) Optimised eigenvalue spectrum.

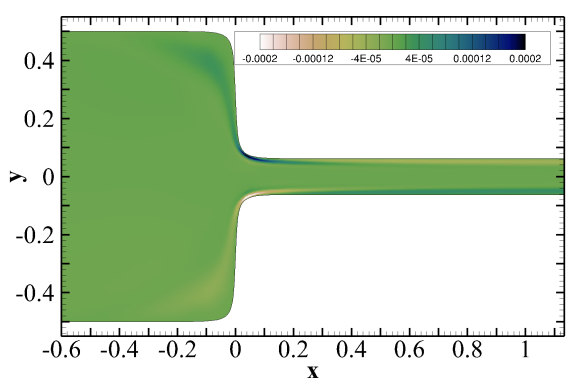

(d) Optimised eigenmodes (horizontal velocity).

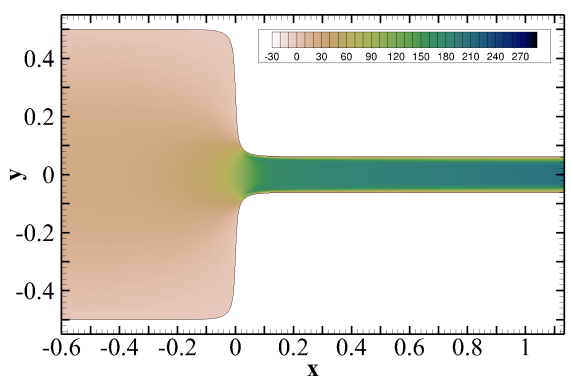

(f) Optimised base flow (horizontal velocity).

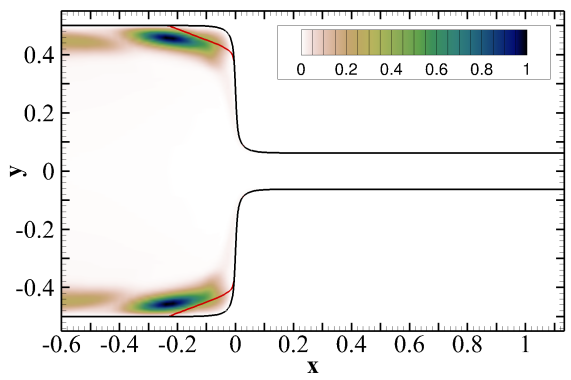

(h) Optimised structural sensitivity.

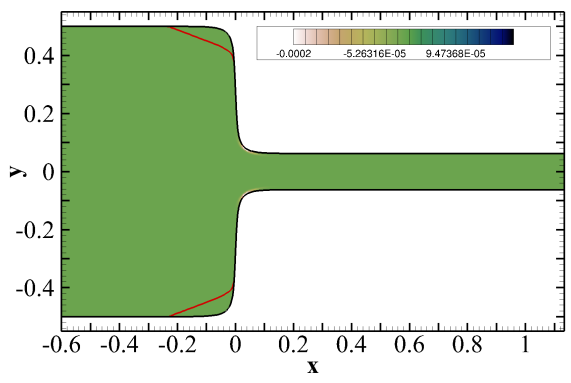

(j) Optimised lift-up perturbation: $u v d U / d y$.

Figure 18: Original and optimised geometries for contraction ratio $C=8$ : eigenvalue spectra, eigenmodes, base flows, structural sensitivities and lift-up perturbation kinetic energy terms. Red lines indicate the edge of recirculation regions. 


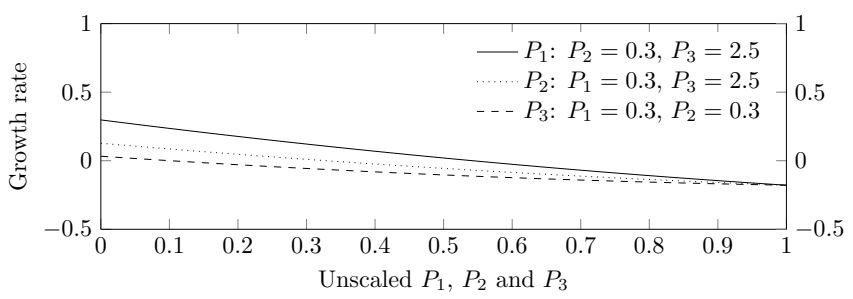

(a) $C=2$.

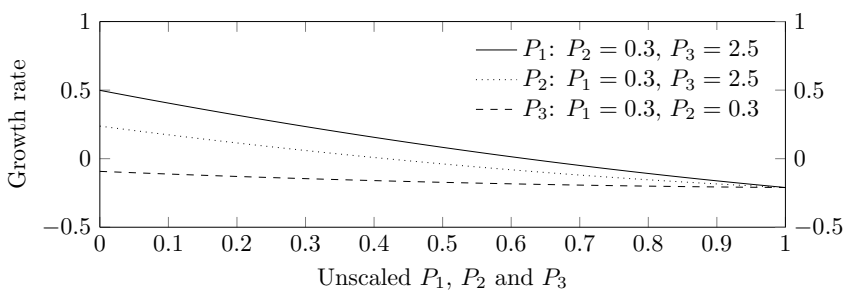

(b) $C=4$.

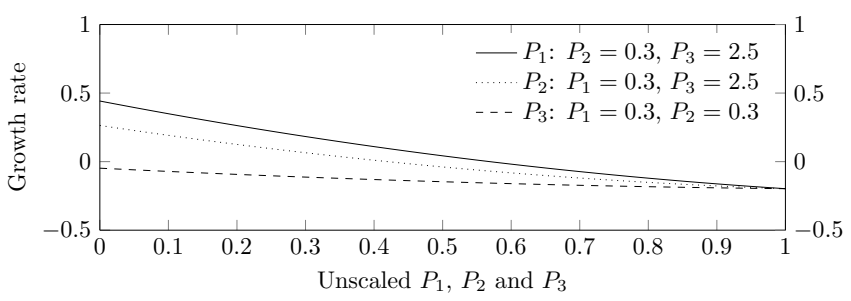

(c) $C=8$.

Figure 19: Parameter-sensitivity of the growth rate to variations in the geometric parameters $\boldsymbol{p}=\left(p_{1}, p_{2}, p_{3}\right)$, near the optimised configuration.

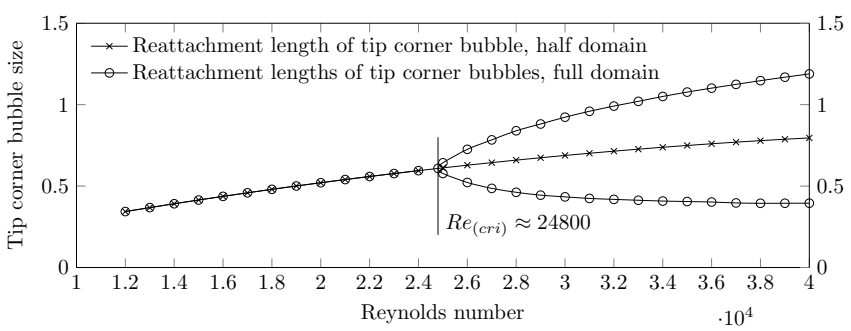

(a) $C=2$.

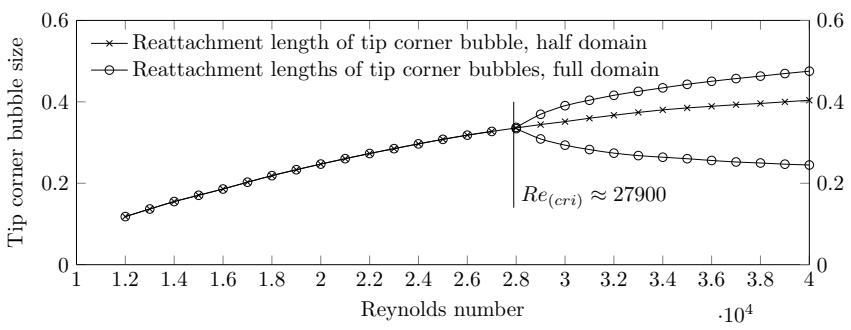

(b) $C=4$.

Figure 20: Bifurcation diagram of the optimised geometries for contraction ratios $C=2$ and 4 , illustrated using the reattachment lengths $L_{3}$ of downstream tip corner eddy for varying Reynolds numbers.
Table X: Drag reduction through shape optimisation.

\begin{tabular}{cccc}
\hline \hline$C$ & $R e_{\text {opt }}$ & $D_{\text {original }}$ & $D_{\text {optimised }}$ \\
\hline 2 & 3200 & 0.116 & 0.042 \\
4 & 1500 & 0.489 & 0.133 \\
8 & 1300 & 1.207 & 0.403 \\
\hline \hline
\end{tabular}

\section{The effect of optimisation on the drag reduction}

We can assess the effect of optimisation through the drag, calculated by integrating the forces on all walls. When the Reynolds number is below the critical value, the drag of the full domain channel is exactly the same as twice the drag in the half domain. However, when the Reynolds number is above the critical value, the drag of the full domain channel becomes greater than twice the drag in the half domain. If the Reynolds number is increased, then the ratio between the full domain drag and the doubled half domain drag is also increased.

We choose the channel with contraction ratio $C=4$ as an example to show the relation between drag coefficient and Reynolds number. As shown in Fig. 22, the drag coefficient of both full domain $\left(D_{f}\right)$ and the drag coefficient of doubled half domain $\left(D_{h}\right)$ reduce when the Reynolds number is increasing. Note that $D_{f} / D_{h}=1$ when the Reynolds number is below the critical value. However, when the Reynolds number is above $R e_{(c r i)}=1420$, the $D_{f} / D_{h}$ ratio becomes greater than 1 and grows exponentially as the Reynolds number increases.

Then we compare in Table $X$ the drag coefficient before and after the optimisation for the three contraction ratios to find that drag can indeed be greatly reduced by eliminating the bifurcation at the optimisation Reynolds number $\left(R e_{o p t}\right)$. The drag coefficients are reduced by $64 \%, 73 \%$ and $67 \%$ for contraction ratios $C=2, C=4$ and $C=8$, respectively.

\section{Three-dimensional Verification on the Optimised Configuration}

Previously, we have presented the stablisation of the twodimensional bifurcation by shape optimisation, and showed that the critical Reynolds number can be greatly increased. In this section, we confirm that these results are still valid when considering three-dimensional flows. To this aim, we proceed to examine whether the two-dimensional optimised shape stabilises the three-dimensional configurations, both original and optimised configurations. In this section, we take contraction ratio $C=4$ as an example to examine the optimisation results in three dimensions.

First, we examine the effect of different spanwise channel lengths on the bubble size. Previous studies by Guevel et al. ${ }^{13}$, Chiang, Sau, and Hwang ${ }^{15}$ have shown that the critical value of Reynolds number and the sizes of the bubbles near the salient corners can depend on the spanwise size, for both contraction channels and expansion channels, by examining the bubble sizes with different spanwise lengths of the channel, using walls as boundary conditions on the two side 


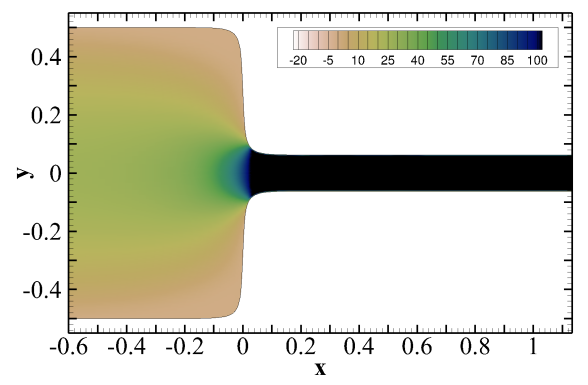

(a) $v_{x}$.

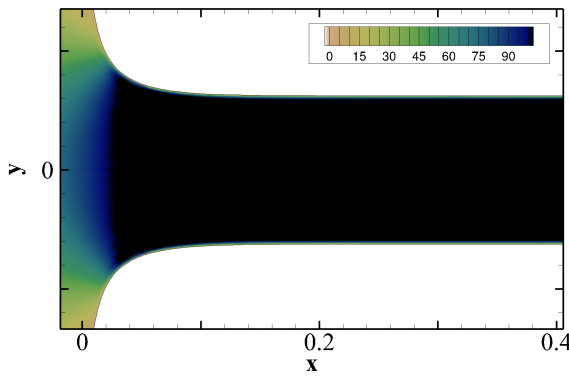

(b) $v_{x}$, zoomed in.

Figure 21: Flow field in the optimised geometry, $C=8$.

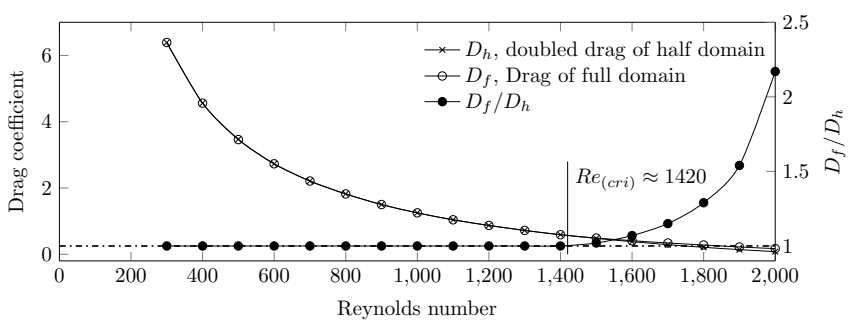

Figure 22: The relation between drag coefficient and Reynolds number.

Table XI: $L_{3}$ against spanwise size, $R e=1600, C=4$.

\begin{tabular}{cc}
\hline \hline Span & $L_{3}$ \\
\hline two-dimensional & 0.648754 \\
$0.4 \pi$ & 0.672116 \\
$0.8 \pi$ & 0.672113 \\
$1.2 \pi$ & 0.672113 \\
$1.6 \pi$ & 0.672114 \\
$2.0 \pi$ & 0.672114 \\
\hline \hline
\end{tabular}

spanwise boundaries.

However, Guevel et al. showed that the bubbles sizes conveyed asymptotically to the two-dimensional sizes as the spanwise length increased, suggesting that three-dimensional flows with periodic boundary conditions can lead to results almost identical to two-dimensional. To confirm this, in the present study, we impose periodic boundary conditions on the two spanwise boundaries. We verify the ability of the periodic boundary condition in representing the channel with an infinite span, by comparing the results with different spanwise lengths.

The sizes of the corner bubbles with different spanwise size is shown in Table XI, with half domain original geometry configuration and contraction ratio $C=4$ at Reynolds number 1600 . We can see that the size of the bubble in twodimensional and three-dimensional simulations are slightly different, and the bubble sizes are bigger in three-dimensional simulations than that in two-dimensional simulations. It is important to note here that when considering in the simulations
Table XII: $L_{3}$ against spanwise size, $R e=1600, C=4$.

\begin{tabular}{cccc}
\hline \hline Case & span & $\left|w_{\max }\right|(\mathrm{m} / \mathrm{s})$ & $L_{3}$ (upper, lower) \\
\hline Original, $R e=2000$ & two-dimensional & 0.0 & $0.854,0.305$ \\
& $2.0 \pi$ & $7.55 \times 10^{-7}$ & $0.895,0.312$ \\
& $4.0 \pi$ & $3.63 \times 10^{-6}$ & $0.895,0.311$ \\
Optimised, $R e=30000$ two-dimensional & 0.0 & $0.391,0.295$ \\
& $2.0 \pi$ & $1.14 \times 10^{-6}$ & $0.444,0.228$ \\
$4.0 \pi$ & $4.90 \times 10^{-9}$ & $0.444,0.228$ \\
\hline \hline
\end{tabular}

of different spanwise lengths, the bubble sizes do not vary. We conclude that when using periodic boundary conditions, there are no important three-dimensional effects affecting the bubbles sizes as suggested in Ref. 13.

Subsequently, we choose $2.0 \pi$ and $4.0 \pi$ as the spanwise size of the channel (wave number $\beta=\frac{2 \pi}{s p a n}=1$ and 2) and check if there exist a three-dimensional bifurcation occurring below the critical Reynolds number for the two-dimensional bifurcation. We take contraction ratio $C=4$, full domain, $R e=2000$ as an example and show in Table XII, that the maximum spanwise component of velocity, $\left|w_{\max }\right|$, is very insignificant, and does not affect the bubble sizes.

We then examine the flow in three dimensions to confirm the effect of optimisation on stabilising the flows in the contraction channels. In Fig. 23, we show the bifurcation diagrams for the three-dimensional original geometry with contraction ratio $C=8$ and with a span of $2 \pi$ and optimised geometry with spans of $2 \pi$ and $4 \pi$. We can see in Fig. 23a that the critical Reynolds number of bifurcation is 1370 which is slightly smaller than that in two-dimensional original geometry (shown in red in Fig. 23a). As shown in Figs. 23b and 23c, the critical Reynolds numbers are 23900 and 23700, which are similar to each other and also slightly smaller than that in two-dimensional optimised geometry (shown in Fig. 20b). Our results suggest that the three-dimensional modes demonstrated in former studies Guevel et al. ${ }^{13}$, Chiang, Sau, and Hwang ${ }^{15}$ are caused by defining the boundary conditions on the spanwise boundaries as walls.

We conclude that the optimisation performed in twodimensional that enhances the $R e_{c r i}$ is still valid for threedimensional flow since no three-dimensional effects neglect 


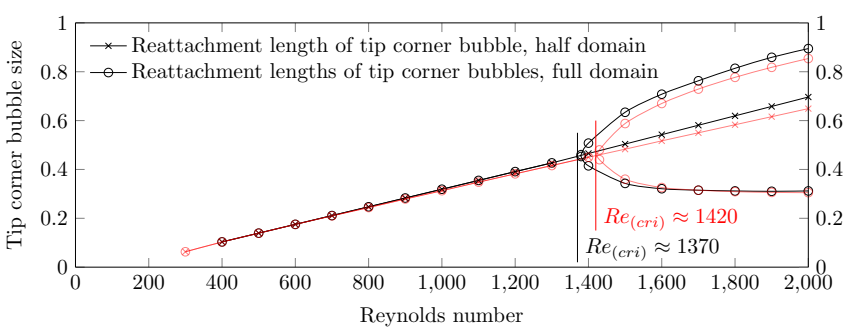

(a) Original geometry, $\operatorname{span}=2 \pi$, (red for 2D).

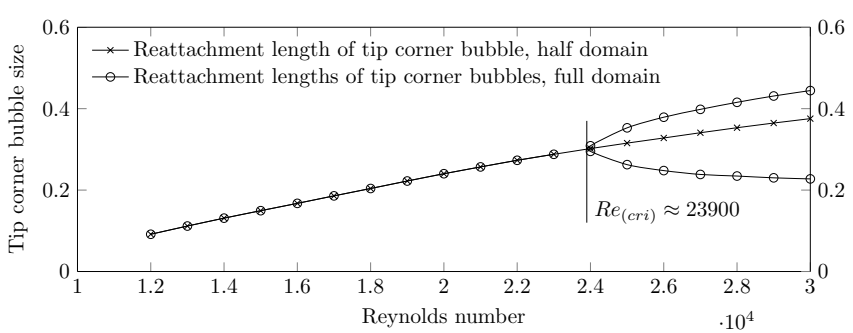

(b) Optimised geometry, span $=2 \pi$.

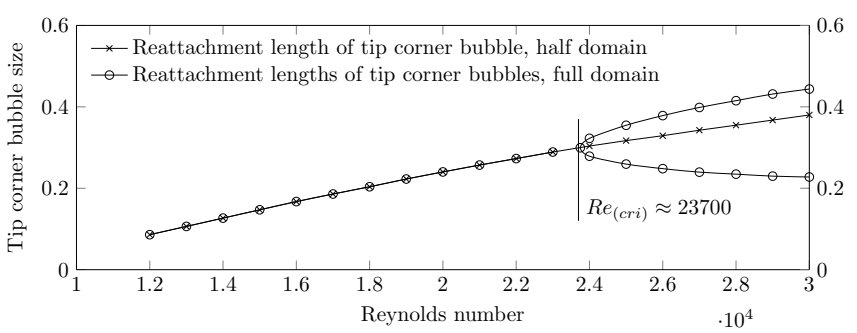

(c) Optimised geometry, $\operatorname{span}=4 \pi$.

Figure 23: Verification of the results with three-dimensional domain.

the advantages of the optimisation.

\section{E. Caveats on Turbulent Flows}

We have presented an optimisation of the contraction channel based on stability analysis that enables a very important increase in the critical Reynolds number that controls the onset asymmetric flow. Additionally, we have shown that the benefits of the two-dimensional optimised geometry hold when considering three-dimensional flows, since no additional three-dimensional bifurcations dominate.

However, the very important increase in critical Reynolds has to be analysed with caution, since other physical mechanisms (e.g. transition due to non-normal transient growth ${ }^{10}$ ) could change the limiting Reynolds here presented and have not been considered in our study. It may be argued that laminar recirculating bubbles can act as noise amplifier, triggering turbulence transition. Therefore, delaying their appearance (avoiding bifurcations) can delay transition to turbulence. Nevertheless, this is only a particular scenario and other mechanisms (e.g. bypass transition due to non-normal transient growth) can lead to early transition.

In fact, it is likely that transition to turbulence could develop at Reynolds numbers above the original critical Reynolds (not optimised geometry) and below the critical Reynolds of the optimised geometries. Analyses of such features remain out of the scope of this work, but in any case neglect the enhancements in delaying asymmetries showed here.

\section{CONCLUSIONS}

The following bullet points summarise the main findings included in this work.

- Computational investigations have been performed to study flow bifurcation in the symmetric planar contraction channel. Flow simulations at different Reynolds numbers for three contraction ratios $(C=2,4,8)$ have been performed and confirm the presence of pitchfork bifurcations, controlled by an asymmetric global mode. Critical Reynolds numbers for all bifurcations are included.

- Stability analysis on the contraction channels relate the eigenmodes responsible for the flow bifurcations to the onset of asymmetries. The instabilities are explained by the lift-up mechanism.

- An optimisation method based on stability analysis is developed for investigations on suppressing the modes responsible for asymmetries. The method consists of an optimiser, a dynamic updating surrogate model, a geometry parametrisation method, a base flow simulator, a stability analysis tool, an eigenmode tracking scheme and a mesh deformation module. This procedure is applied to suppress the asymmetric mode through small shape deformations.

- The optimised geometries show rounded corners and minimise the lift-up physical mechanisms responsible for the instability governing the asymmetric flow. We show that very small modification in the geometry lead to a complete change in the stability, increasing the critical Reynolds numbers of the bifurcations.

- We show that the critical Reynolds number of the original geometry can be increased significantly when optimising the shape. The optimised geometries are stable for Reynolds numbers well beyond the original values: 7.9 and 20.9 times higher for contraction ratios $C=2$ and 4 respectively. The tip corner bubbles are entirely eliminated for contraction ratio $C=8$ for Reynolds numbers as high as $4 \times 10^{6}$.

- The optimised geometry configuration is investigated in three dimensions and confirm that the enhanced stability obtained through the two-dimensional optimisation hold in three-dimensional flows.

- At the optimisation Reynolds numbers $\left(R e_{\text {opt }}\right)$ for each contraction ratios, the drag coefficients are also greatly reduced, by $64 \%, 73 \%$ and $67 \%$ for contraction ratios $C=2,4$ and 8 , respectively. 
- In the future, the gradient information of the growth rate to the shape parameters will guide the optimisation investigation through a gradient based approach.

\section{ACKNOWLEDGMENTS}

The authors acknowledge the financial support of SSeMID: Stability and Sensitivity Methods for Industrial Design funded by European Union's Horizon 2020 research and innovation program under the Marie Skłodowska-Curie grant agreement No. 675008.

\section{Appendix A: Linear stability analysis}

Starting with the system

$$
\mathscr{M} \frac{\partial \boldsymbol{q}}{\partial t}=\mathscr{F}(\boldsymbol{q})
$$

we introduce the state variable decomposition $\boldsymbol{q}=\overline{\boldsymbol{q}}+\boldsymbol{q}^{\prime}$, to obtain

$$
\mathscr{M}\left(\frac{\partial \overline{\boldsymbol{q}}}{\partial t}+\frac{\partial \boldsymbol{q}^{\prime}}{\partial t}\right)=\mathscr{F}\left(\overline{\boldsymbol{q}}+\boldsymbol{q}^{\prime}\right)
$$

To linearise the non-linear operator $\mathscr{F}\left(\overline{\boldsymbol{q}}+\boldsymbol{q}^{\prime}\right)$, we use Taylor series (around $\overline{\boldsymbol{q}}$ ):

$$
\mathscr{F}\left(\overline{\boldsymbol{q}}+\boldsymbol{q}^{\prime}\right)=\mathscr{F}(\overline{\boldsymbol{q}})+\left.\frac{\partial \mathscr{F}(\boldsymbol{q})}{\partial \boldsymbol{q}}\right|_{\overline{\boldsymbol{q}}} \boldsymbol{q}^{\prime}+\mathscr{O}\left(\boldsymbol{q}^{2}\right) .
$$

Neglecting second order terms $\left(\mathscr{O}\left(\boldsymbol{q}^{\prime 2}\right)\right)$, and replacing Eq. A3 into Eq. A2, we obtain a linearised version of Eq. A2.

$$
\mathscr{M}\left(\frac{\partial \overline{\boldsymbol{q}}}{\partial t}+\frac{\partial \boldsymbol{q}^{\prime}}{\partial t}\right)=\mathscr{F}(\overline{\boldsymbol{q}})+\left.\frac{\partial \mathscr{F}(\boldsymbol{q})}{\partial \boldsymbol{q}}\right|_{\overline{\boldsymbol{q}}} \boldsymbol{q}^{\prime}
$$

Finally, we may subtract the base flow equation: $\mathscr{M} \frac{\partial \overline{\mathbf{q}}}{\partial \mathbf{t}}=$ $\mathscr{F}(\overline{\mathbf{q}})$, to arrive at the final expression for the time evolution of perturbations:

$$
\mathscr{M} \frac{\partial \boldsymbol{q}^{\prime}}{\partial t}=\left.\frac{\partial \mathscr{F}(\boldsymbol{q})}{\partial \boldsymbol{q}}\right|_{\overline{\boldsymbol{q}}} \boldsymbol{q}^{\prime}
$$

\section{REFERENCES}

${ }^{1}$ S. Dennis and F. Smith, "Steady flow through a channel with a symmetrical constriction in the form of a step," Proc. R. Soc. Lond. A 372, 393-414 (1980).

${ }^{2} \mathrm{R}$. Hunt, "The numerical solution of the laminar flow in a constricted channel at moderately high Reynolds number using Newton iteration," International Journal for Numerical Methods in Fluids 11, 247-259 (1990).

${ }^{3}$ D. M. Hawken, P. Townsend, and M. F. Webster, "Numerical simulation of viscous flows in channels with a step," Computers and Fluids 20, 59-75 (1991).

${ }^{4}$ F. Durst, W. F. Schierholz, and A. M. Wunderlich, "Experimental and Numerical Investigations of Plane Duct Flows With Sudden Contraction," Journal of Fluids Engineering 109, 376-373 (1987).
${ }^{5}$ W. Cherdron, F. Durst, and J. H. Whitelaw, "Asymmetric flows and instabilities in symmetric ducts with sudden expansions," Journal of Fluid Mechanics 84, 13 (1978).

${ }^{6}$ I. J. Sobey and P. G. Drazin, "Bifurcations of two-dimensional channel flows," Journal of Fluid Mechanics 171, 263-287 (1986).

${ }^{7}$ T. P. Chiang and T. W. H. Sheu, "Bifurcations of Flow Through Plane Symmetric Channel," Journal of Fluids Engineering 124, 444-451 (2002).

${ }^{8}$ P. G. Drazin and W. H. Reid, Hydrodynamic Stability, 2nd ed., Cambridge Mathematical Library (Cambridge University Press, 2004).

${ }^{9}$ R. Wille and H. Fernholz, "Report on the first European Mechanics Colloquium, on the Coanda effect," Journal of Fluid Mechanics 23, 801-819 (1965).

${ }^{10}$ P. J. Schmid and D. S. Henningson, Stability and Transition in Shear Flows (Springer, 2001).

${ }^{11}$ R. M. Fearn, T. Mullin, and A. K. Cliffe, "Nonlinear flow phenomena in a symmetric sudden expansion,” Journal of Fluid Mechanics 211, 595-608 (1990).

${ }^{12}$ A. Fani, S. Camarri, and M. V. Salvetti, "Stability analysis and control of the flow in a symmetric channel with a sudden expansion," Physics of Fluids 24 (2012), 10.1063/1.4745190.

${ }^{13}$ Y. Guevel, T. Allain, G. Girault, and J. M. Cadou, "Numerical bifurcation analysis for 3-dimensional sudden expansion fluid dynamic problem," International Journal for Numerical Methods in Fluids (2018), 10.1002/fld.4478.

${ }^{14}$ A. Shahbani-Zahiri, H. Hassanzadeh, M. M. Shahmardan, and M. Norouzi, "Investigation of pitchfork bifurcation phenomena effects on heat transfer of viscoelastic flow inside a symmetric sudden expansion," Physics of Fluids (2017), 10.1063/1.5009434.

${ }^{15}$ T. P. Chiang, A. Sau, and R. R. Hwang, "Asymmetry and bifurcations in three-dimensional sudden-contraction channel flows," Physical Review E - Statistical, Nonlinear, and Soft Matter Physics (2011), 10.1103/PhysRevE.83.046313.

${ }^{16}$ J. Mizushima and Y. Shiotani, "Transitions and instabilities of flow in a symmetric channel with a suddenly expanded and contracted part,' Journal of Fluid Mechanics 434, 355-369 (2001).

${ }^{17}$ I. Lashgari, O. Tammisola, V. Citro, M. P. Juniper, and L. Brandt, "The planar X-junction flow: stability analysis and control," Journal of Fluid Mechanics 753, 1-28 (2014).

${ }^{18} \mathrm{~S}$. Thomas, T. Ameel, and J. Guilkey, "Mixing kinematics of moderate Reynolds number flows in a T-channel," Physics of Fluids 22, 1-10 (2010).

${ }^{19}$ K. K. Chen, C. W. Rowley, and H. A. Stone, "Vortex dynamics in a pipe T-junction: Recirculation and sensitivity," Physics of Fluids (2015), 10.1063/1.4916343.

${ }^{20}$ B. Laborie, F. Rouyer, D. E. Angelescu, and E. Lorenceau, "On the stability of the production of bubbles in yield-stress fluid using flow-focusing and Tjunction devices," Physics of Fluids (2016), 10.1063/1.4953678.

${ }^{21}$ F. Gallaire, M. Marquillie, and U. Ehrenstein, "Three-dimensional transverse instabilities in detached boundary layers," Journal of Fluid Mechanics 571, 221-233 (2007).

${ }^{22}$ P. Y. Passaggia, T. Leweke, and U. Ehrenstein, "Transverse instability and low-frequency flapping in incompressible separated boundary layer flows: An experimental study," Journal of Fluid Mechanics 703, 363-373 (2012).

${ }^{23}$ D. Barkley, M. G. M. Gomes, and R. D. Henderson, "Three-dimensional instability in flow over a backward-facing step," Journal of Fluid Mechanics , 167-190 (2002).

${ }^{24}$ O. Marquet, D. Sipp, and L. Jacquin, "Sensitivity analysis and passive control of cylinder flow," Journal of Fluid Mechanics 615, 221-252 (2008).

${ }^{25}$ D. Lanzerstorfer and H. C. Kuhlmann, "Global stability of the twodimensional flow over a backward-facing step,” Journal of Fluid Mechanics 693, 1-27 (2011).

${ }^{26} \mathrm{D}$. Rodríguez and V. Theofilis, "Structural changes of laminar separation bubbles induced by global linear instability," Journal of Fluid Mechanics 655, 280-305 (2010).

${ }^{27}$ S. Albensoeder, H. C. Kuhlmann, and H. J. Rath, "Three-dimensional centrifugal-flow instabilities in the lid-driven-cavity problem," Physics of Fluids 13, 121-135 (2001).

${ }^{28}$ S. Haque, I. Lashgari, F. Giannetti, and L. Brandt, "Stability of fluids with shear-dependent viscosity in the lid-driven cavity," Journal of NonNewtonian Fluid Mechanics 173-174, 49-61 (2012). 
${ }^{29}$ P. Luchini and A. Bottaro, "Adjoint Equations in Stability Analysis," Annual Review of Fluid Mechanics 46, 493-517 (2014).

${ }^{30}$ D. C. Hill, "A theoretical approach for analyzing the restabilization of wakes," in AIAA Aersoapce Science Meeting Exhib. 30th, Reno, NV, AIAA Pap., Vol. 0067 (1992).

${ }^{31}$ F. Gianetti and P. Luchini, "Structural sensitivity of the first instability of the cylinder wake," Journal of Fluid Mechanics 581, 167-197 (2007).

${ }^{32}$ E. Ferrer, J. de Vicente, and E. Valero, "Low cost 3D global instability analysis and flow sensitivity based on dynamic mode decomposition and high-order numerical tools," International Journal for Numerical Methods in Fluids 76, 169-184 (2014).

${ }^{33}$ O. M. F. Browne, G. Rubio, E. Ferrer, and E. Valero, "Sensitivity analysis to unsteady perturbations of complex flows: A discrete approach," International Journal for Numerical Methods in Fluids 76, 1088-1110 (2014).

${ }^{34}$ M. C. Iorio, L. M. González, and E. Ferrer, "Direct and adjoint global stability analysis of turbulent transonic flows over a NACA0012 profile," International Journal for Numerical Methods in Fluids 76, 147-168 (2014).

${ }^{35}$ L. Gonzalez, E. Ferrer, and H. Diaz-Ojeda, "Onset of three-dimensional flow instabilities in lid-driven circular cavities," Physics of Fluids 29, 064102 (2017).

${ }^{36}$ E. Ferrer, O. M. Browne, and E. Valero, "Sensitivity analysis to control the far-wake unsteadiness behind turbines," Energies 10 (2017).

${ }^{37}$ D. Sipp, O. Marquet, P. Meliga, and A. Barbagallo, "Dynamics and control of global instabilities in open flows: a linearized approach," Applied Mechanical reviews 63, 1-26 (2010).

${ }^{38}$ J. Périaux, "Optimization Methods and Tools for Multi Objectives DESIGN using Evolutionary Algorithms and Game strategies," , 1-44 (2014).

${ }^{39}$ L. Huyse, S. L. Padula, R. M. Lewis, and W. Li, "Probabilistic approach to free-form airfoil shape optimization under uncertainty," AIAA Journal 40, 1764-1772 (2002).

${ }^{40}$ J. O. Pralits and A. Hanifi, "Optimization of steady suction for disturbance control on infinite swept wings," Physics of Fluids (2003), 10.1063/1.1597684.

${ }^{41}$ T. L. Flinois and T. Colonius, "Optimal control of circular cylinder wakes using long control horizons," Physics of Fluids (2015), 10.1063/1.4928896.

${ }^{42}$ D. Xiao and G. Papadakis, "Nonlinear optimal control of bypass transition in a boundary layer flow," Physics of Fluids (2017), 10.1063/1.4983354.

${ }^{43}$ N. V. Queipo, R. T. Haftka, W. Shyy, T. Goel, R. Vaidyanathan, and P. Kevin Tucker, "Surrogate-based analysis and optimization," Progress in Aerospace Sciences 41, 1-28 (2005).

${ }^{44}$ C. Mettot, F. Renac, and D. Sipp, "Computation of eigenvalue sensitivity to base flow modifications in a discrete framework: Application to open-loop control," Journal of Computational Physics 269, 234 - 258 (2014).

${ }^{45}$ G. J. Chandler, M. P. Juniper, J. W. Nichols, and P. J. Schmid, “Adjoint algorithms for the Navier-Stokes equations in the low Mach number limit,"
Journal of Computational Physics 231, 1900-1916 (2012).

${ }^{46}$ D. Barkley, H. M. Blackburn, and S. J. Sherwin, "Direct optimal growth analysis for timesteppers," International Journal for Numerical Methods in Fluids 57, 1435-1458 (2008).

${ }^{47}$ J.-M. Chomaz, "Global Instabilities in Spatially Developing Flows: NonNormality and Nonliearity," Annual Review of Fluid Mechanics 37, 357 392 (2005).

${ }^{48} \mathrm{O}$. Marquet and L. Lesshafft, "Identification of contributing flow regions for linear and nonlinear flow instabilities," arXiv preprint (2015), arXiv: 1508.07620.

${ }^{49}$ J. O. Pralits, L. Brandt, and F. Giannetti, "Instability and sensitivity of the flow around a rotating circular cylinder," Journal of Fluid Mechanics (2010), 10.1017/S0022112009993764.

${ }^{50}$ P. R. Amestoy, I. S. Duff, J.-Y. L'Excellent, and J. Koster, "A Fully Asynchronous Multifrontal Solver Using Distributed Dynamic Scheduling," SIAM Journal on Matrix Analysis and Applications 23, 15-41 (2001).

${ }^{51}$ M. Planitz and E. Anderson, "LAPACK Users Guide," The Mathematical Gazette 79, 210 (1995).

${ }^{52} \mathrm{~T}$. Hawa and Z. Rusak, "The dynamics of a laminar flow in a symmetric channel with a sudden expansion," Journal of Fluid Mechanics 436, 283320 (2001).

${ }^{53} \mathrm{~L}$. Brandt, "The lift-up effect: The linear mechanism behind transition and turbulence in shear flows," European Journal of Mechanics - B/Fluids 47, 80 - 96 (2014), Enok Palm Memorial Volume.

${ }^{54}$ M. Kumar, M. Husian, N. Upreti, and D. Gupta, "Genetic Algorithm: Review and Application," International Journal of Information Technology and Knowledge Management 2, 451-454 (2010).

${ }^{55}$ L. Davis, ed., Handbook of Genetic Algorithms (Van Nostrand Reinhold, 1991).

${ }^{56}$ J. Samareh, "Aerodynamic shape optimization based on free-form deformation," in 10th AIAA/ISSMO Multidisciplinary Analysis and Optimization Conference, edited by A. I. of Aeronautics and Astronautics (2004).

${ }^{57}$ Z.-H. Han and K.-S. Zhang, "Surrogate-Based Optimization," Real-World Applications of Genetic Algorithms, 343-362 (2008).

${ }^{58}$ T. J. Mackman and C. B. Allen, "Comparison of Parameter Tuning Strategies for RBF Surrogate Models with Adaptive Sampling," Evaluation , 1-14 (2011).

${ }^{59}$ R. Yondo, E. Andrés, and E. Valero, "A review on design of experiments and surrogate models in aircraft real-time and many-query aerodynamic analyses," Progress in Aerospace Sciences 96, 23 - 61 (2018).

${ }^{60}$ A.-H. Jiang, X.-C. Huang, Z.-H. Zhang, J. Li, Z.-Y. Zhang, and H.-X. Hua, "Uncertainty quantification of squeal instability via surrogate modelling," Mechanical Systems and Signal Processing 24, 2947-2960 (2010).

${ }^{61} \mathrm{O}$. Tammisola, "Optimal wavy surface to suppress vortex shedding using second-order sensitivity to shape changes," European Journal of Mechanics, B/Fluids (2017), 10.1016/j.euromechflu.2016.12.006, arXiv:1602.03378. 